\title{
The Opportunity Offered by the ESSnuSB Project to Exploit the Larger Leptonic CP Violation Signal at the Second Oscillation Maximum and the Requirements of This Project on the ESS Accelerator Complex
}

\author{
E. Wildner, ${ }^{1}$ E. Baussan, ${ }^{2}$ M. Blennow, ${ }^{3}$ M. Bogomilov, ${ }^{4}$ \\ A. Burgman, ${ }^{5}$ E. Bouquerel, ${ }^{2}$ C. Carlile, ${ }^{6}$ J. Cederkäll, ${ }^{5}$ P. Christiansen, ${ }^{5}$ \\ P. Cupial, ${ }^{7}$ H. Danared, ${ }^{8}$ M. Dracos, ${ }^{2}$ T. Ekelöf, ${ }^{6}$ M. Eshraqi, ${ }^{8}$ \\ R. Hall-Wilton, ${ }^{8}$ J.-P. Koutchouk, ${ }^{1,6}$ M. Lindroos, ${ }^{8}$ M. Martini, ${ }^{1}$ \\ R. Matev, ${ }^{4}$ D. McGinnis, ${ }^{8}$ R. Miyamoto, ${ }^{8}$ T. Ohlsson, ${ }^{3}$ H. Öhman, ${ }^{6}$ \\ M. Olvegård, ${ }^{6}$ R. Ruber, ${ }^{6}$ H. Schönauer, ${ }^{1}$ J. Y. Tang, ${ }^{9}$ R. Tsenov, ${ }^{4}$ \\ G. Vankova-Kirilova, ${ }^{4}$ and N. Vassilopoulos ${ }^{9}$ \\ ${ }^{1}$ CERN, 1211 Geneva 23, Switzerland \\ ${ }^{2} I P H C$, Université de Strasbourg, CNRS/IN2P3, 67037 Strasbourg, France \\ ${ }^{3}$ Department of Theoretical Physics, School of Engineering Sciences, KTH Royal Institute of Technology, \\ AlbaNova University Center, 10691 Stockholm, Sweden \\ ${ }^{4}$ Department of Atomic Physics, St. Kliment Ohridski University of Sofia, 1164 Sofia, Bulgaria \\ ${ }^{5}$ Department of Physics, Lund University, P.O. Box 118, 22100 Lund, Sweden \\ ${ }^{6}$ Department of Physics and Astronomy, Uppsala University, P.O. Box 516, 75120 Uppsala, Sweden \\ ${ }^{7}$ AGH University of Science and Technology, Aleja Mickiewicza 30, 30-059 Krakow, Poland \\ ${ }^{8}$ European Spallation Source, ESS ERIC, P.O. Box 176, 22100 Lund, Sweden \\ ${ }^{9}$ Institute of High Energy Physics, CAS, Beijing 100049, China
}

Correspondence should be addressed to E. Wildner; elena.wildner@cern.ch and T. Ekelöf; tord.ekelof@physics.uu.se

Received 3 September 2015; Revised 18 December 2015; Accepted 29 December 2015

Academic Editor: Leslie Camilleri

Copyright ( 2016 E. Wildner et al. This is an open access article distributed under the Creative Commons Attribution License, which permits unrestricted use, distribution, and reproduction in any medium, provided the original work is properly cited. The publication of this article was funded by $\mathrm{SCOAP}^{3}$.

\footnotetext{
The European Spallation Source (ESS), currently under construction in Lund, Sweden, is a research center that will provide, by 2023, the world's most powerful neutron source. The average power of the proton linac will be $5 \mathrm{MW}$. Pulsing this linac at higher frequency will make it possible to raise the average total beam power to $10 \mathrm{MW}$ to produce, in parallel with the spallation neutron production, a very intense neutrino Super Beam of about $0.4 \mathrm{GeV}$ mean neutrino energy. This will allow searching for leptonic $\mathrm{CP}$ violation at the second oscillation maximum where the sensitivity is about 3 times higher than at the first. The ESS neutrino Super Beam, ESSnuSB operated with a $2.0 \mathrm{GeV}$ linac proton beam, together with a large underground Water Cherenkov detector located at $540 \mathrm{~km}$ from Lund, will make it possible to discover leptonic CP violation at $5 \sigma$ significance level in $56 \%$ (65\% for an upgrade to $2.5 \mathrm{GeV}$ beam energy) of the leptonic $\mathrm{CP}$-violating phase range after 10 years of data taking, assuming a $5 \%$ systematic error in the neutrino flux and $10 \%$ in the neutrino cross section. The paper presents the outstanding physics reach possible for CP violation with ESSnuSB obtainable under these assumptions for the systematic errors. It also describes the upgrade of the ESS accelerator complex required for ESSnuSB.
} 


\section{Overview}

An ultimate goal of the long baseline neutrino experiments now being planned is the discovery and high precision measurement of the leptonic CP violation through measurement of the $v_{\mu} \rightarrow v_{e}$ oscillation probability. Such a discovery and measurement would shed new light on the fundamental problem of how to explain the dominance of matter over antimatter in the Universe. The discovery and measurement in 2012 of a nonzero value as large as ca. $9^{\circ}$ of the neutrino mixing angle $\theta_{13}$, corresponding to a value of $\sin ^{2} 2 \theta_{13}$ of ca. 0.095 [1-4], confirmed the possibility of discovering and measuring a nonzero value of the Dirac leptonic CP violating angle $\delta_{\mathrm{CP}}$. Before 2012 a significantly smaller value for $\theta_{13}$ was assumed when planning for future long base neutrino experiments. See, for example, [5], in which a range of values for $\sin ^{2} 2 \theta_{13}$ between 0.01 and 0.09 are used, with 0.04 as the standard value, to evaluate the performance of a proposed experiment. The large value of $\theta_{13}$ implies that the sensitivity to $\mathrm{CP}$ violation is three times higher at the second neutrino oscillation maximum as compared to the first.

The European Spallation Source (ESS) [6] under construction in Lund, Sweden, since the fall of 2014, is a research center that will have the world's most powerful neutron source. It is based on a $2.0 \mathrm{GeV}$ superconducting linac (with space available for an upgrade to the originally planned energy of $2.5 \mathrm{GeV}$ ), providing $2.86 \mathrm{~ms}$ long proton pulses at $14 \mathrm{~Hz}$ for the spallation neutron facility with $5 \mathrm{MW}$ average power on target (Figure 1). By pulsing the linac at higher frequency, additional beam pulses can be interleaved to provide a total average beam power of $10 \mathrm{MW}$. The extra pulses, providing $1.5 \cdot 10^{16}$ protons per second on target, corresponding to $2.7 \cdot 10^{23}$ protons on target per year, can be used to obtain a neutrino beam of unprecedented intensity. The uniquely high intensity of the ESS linac allows for sufficient event statistics to be collected with a Megaton neutrino detector positioned at the second neutrino oscillation maximum, where the relative $\mathrm{CP}$ violation sensitivity is about three times higher than at the first maximum, where the DUNE experiment in the USA [7] and the Hyper$\mathrm{K}$ experiment in Japan [8] have their respective detectors positioned.

In a first publication [9] the European Spallation Source neutrino Super Beam (ESSnuSB) collaboration proposes searching for leptonic $\mathrm{CP}$ violation by making use of the Super Beam described above and a Megaton water Cherenkov detector placed in the $1200 \mathrm{~m}$ deep Garpenberg mine located at a distance of $540 \mathrm{~km}$ from the neutrino source in Lund, near the second neutrino oscillation maximum. In the present paper we report on some further progress made in the study of the ESSnuSB project, in particular regarding its performance for $\mathrm{CP}$ violation discovery and measurement, the proton accumulator ring, and the Near Detector. Table 1 shows an overview of the parameters of the facility. The ESSnuSB Design Study is taking advantage of many of the results obtained in the FP7 Design Study EUROnu [10] on future neutrino facilities. The results from the now terminated EUROnu project of the study of the $4.5 \mathrm{GeV} / 5 \mathrm{MW}$ neutrino Super Beam from the CERN Superconducting Proton Linac
TABLE 1: Main parameters of the ESSnuSB neutrino facility.

\begin{tabular}{lc}
\hline Parameter & Value \\
\hline Average neutrino energy & $0.36 \mathrm{GeV}$ \\
Baseline & $540 \mathrm{~km}$ \\
Detector technology & Water Cherenkov \\
Fiducial volume & $500 \mathrm{kt}$ \\
PMTs & $240 \mathrm{k} 8^{\prime \prime}$ \\
10 -year reach $(2 \nu+8 \bar{\nu})$ & $60 \%$ of $\delta_{\mathrm{CP}}$ range $(5 \sigma)$ \\
$L / E(2$ nd oscillation max. $)$ & $1500 \mathrm{~km} / \mathrm{GeV}$ \\
\hline
\end{tabular}

TABLE 2: Current main parameters of the ESS linac.

\begin{tabular}{lc}
\hline Parameter & Value \\
\hline Average beam power & $5 \mathrm{MW}$ \\
Ion kinetic energy & $2 \mathrm{GeV}$ \\
Average macro pulse current & $62.5 \mathrm{~mA}$ \\
Average macro pulse length & $2.86 / 4 \mathrm{~ms}$ \\
Pulse repetition rate & $14 \mathrm{~Hz}$ \\
Maximum accelerating cavity surface field & $45 \mathrm{MV} / \mathrm{m}$ \\
Linac length & $352.5 \mathrm{~m}$ \\
Reliability & $95 \%$ \\
Annual operating period & $5000 \mathrm{~h}$ \\
\hline
\end{tabular}

SPL $[11,12]$ and of the MEMPHYS large water Cherenkov detector in the Fréjus tunnel have served as very useful references in the ESSnuSB Design Study $[13,14]$.

The purpose of ESS is the production and use of spallation neutrons. The use of the ESS linac for neutron and neutrino production simultaneously, not reducing neutron production, will decrease considerably the cost of the proposed neutrino project as compared to constructing a dedicated proton driver for generating the neutrino beam. The current main parameters of the ESS proton driver are listed in Table 2.

With presently available technology, the horn-type hadron collector cannot handle $2.86 \mathrm{~ms}$ long pulses due to the excessive ohmic heating of the magnet-system currentleads. Therefore, the linac pulse has to be accumulated in a multiturn injection storage ring that can deliver, through single turn extraction, pulses of a few $\mu$ s length to the neutrino production target and horn assembly. There is space available on the ESS site to implement such a proton accumulator and its transfer lines from the linac to the target station as well as for the implementation of the target station itself and a Near Detector. To inject protons from the linac into the accumulator with satisfactory efficiency, charge exchange injection will be necessary. Therefore, the ESS linac has to be further equipped such that it can be used to accelerate $\mathrm{H}^{-}$pulses of the same length and intensity as the proton pulses.

A preliminary study of the modifications of the ESS linac that are required to allow simultaneous acceleration of $\mathrm{H}^{+}$ and $\mathrm{H}^{-}$ions at an average power of $5+5 \mathrm{MW}$ has been made [15]. It is proposed that some of these modifications be made already during the current linac construction phase, such that it will later be possible to upgrade the linac with only minor interventions during the operation of the linac for 


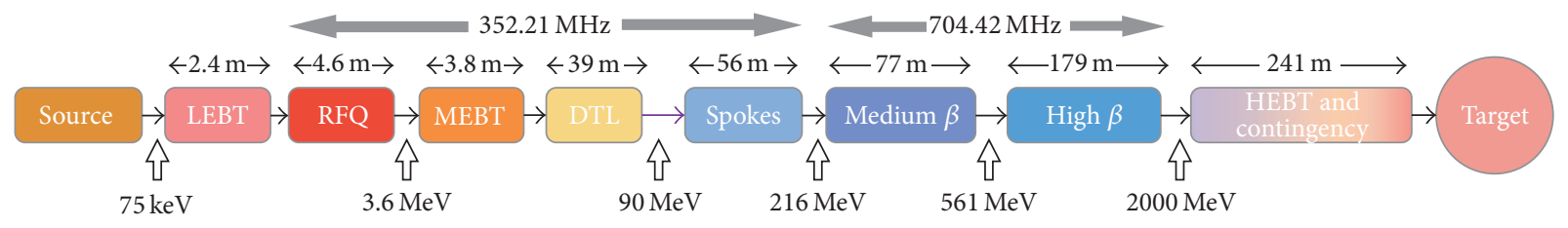

FIgURE 1: The ESS superconducting linac.

spallation neutron production. These modifications, which will increase the initial construction cost of the linac by ca. $10 \%$, will significantly reduce the cost of the subsequent upgrade of the linac to enable the production of a neutrino beam.

The proposed upgrade of the accelerator complex will significantly increase the potential and options also for other future developments of the ESS. One example of this is that the accumulator studies aim at a design allowing the accumulator to satisfy the requirements for the production of both short neutrino pulses of a few $\mu$ s length through single turn extraction and short neutron pulses of the order of $100 \mu \mathrm{s}$ length through multiturn extraction, thus also providing, as a future option, the production of short, uniquely intense neutron pulses [16].

The EUROnu studies identified some key elements of the SPL Super Beam for which further R\&D would be necessary, such as the proton target, the hadron collector, and its pulse generator. These items will be further studied to prove their feasibility for the ESS based neutrino project. Simulation studies have resulted in the choice of the $1200 \mathrm{~m}$ deep Garpenberg mine in the Swedish Dalarna county, $540 \mathrm{~km}$ from ESS in Lund, as the optimal location for the Far Neutrino Detector. The Near Detector will contain a water Cherenkov detector and possibly other types of detectors. Results from the European FP7 LAGUNA Design Study [17] provide useful information for the design of the detectors and the needed infrastructure.

\section{The Significant Advantage of Measuring Leptonic CP Violation at the Second Oscillation Maximum}

The following expression describes the probability for the $\nu_{\mu} \rightarrow v_{e}$ oscillation [19]:

$$
\begin{aligned}
P\left(v_{\mu}\right. & \left.\longrightarrow v_{e}\right) \\
= & \sin ^{2} \theta_{23} \sin ^{2} 2 \theta_{13} \sin ^{2}\left(\frac{\Delta_{31} L}{2}\right) \\
& +\cos ^{2} \theta_{23} \sin ^{2} 2 \theta_{12} \sin ^{2}\left(\frac{\Delta_{21} L}{2}\right) \\
& +\widetilde{J} \cos \left(\delta_{\mathrm{CP}}-\frac{\Delta_{31} L}{2}\right) \sin \left(\frac{\Delta_{21} L}{2}\right) \sin \left(\frac{\Delta_{31} L}{2}\right),
\end{aligned}
$$

where $\widetilde{J} \equiv \cos \theta_{13} \sin 2 \theta_{12} \sin 2 \theta_{23} \sin 2 \theta_{13}$ and $\Delta_{i j} \equiv \Delta m_{i j}^{2} /$ $2 E_{v}$. The sign of $\delta_{\mathrm{CP}}$ is the opposite for antineutrinos.

The first two terms in this expression are generally referred to as the "atmospheric" term and the "solar" term, respectively. The third is the " $\mathrm{CP}$ interference" term which is the only term that depends on the CP violating angle $\delta_{\mathrm{CP}}$.

Plots for two different values of the mixing angle, $\theta_{13}=1^{\circ}$ and $\theta_{13}=10^{\circ}$, of the three terms in the $\nu_{\mu} \rightarrow \nu_{e}$ oscillation probability expression are shown in Figure 2 as a function of the variable $L / E$, the ratio between the accelerator-detector distance $L$ (the baseline) and the neutrino energy $E$. The $\mathrm{CP}$ violating term is calculated without the factor $\cos \left(\delta_{\mathrm{CP}}-\right.$ $\left.\Delta_{31} L / 2\right)$; that is, what is shown is the maximum value this term could take on. The first and second neutrino oscillation maxima are clearly seen in the atmospheric term to be at around $L / E=500 \mathrm{~km} / \mathrm{GeV}$ and $1500 \mathrm{~km} / \mathrm{GeV}$.

The information on $\delta_{\mathrm{CP}}$ is thus contained in the $\mathrm{CP}$ interference term. As can be seen in Figure 2(a), for a value of the neutrino mixing angle $\theta_{13}$ that is small, like $1^{\circ}$, which lies within the range of values used in the long baseline neutrino study projects before 2012, the CP interference term at $L / E=$ $500 \mathrm{~km} / \mathrm{GeV}$ is somewhat larger than both the solar and the atmospheric term, whereas at $L / E=1500 \mathrm{~km} / \mathrm{GeV}$ the $\mathrm{CP}$ interference term is much smaller than the dominant solar term. Had the value of $\theta_{13}$ indeed been small, the sensitivity to $\delta_{\mathrm{CP}}$ would thus have been much higher at $L / E=500 \mathrm{~km} / \mathrm{GeV}$ than at $L / E=1500 \mathrm{~km} / \mathrm{GeV}$. This is certainly one reason why some proposed projects, which were designed well before 2012, have their respective detectors placed at a distance from the accelerator approximately corresponding to $L / E=$ $500 \mathrm{~km} / \mathrm{GeV}$, that is, at the first maximum. However, when in $2012 \theta_{13}$ was measured and found to be about $9^{\circ}[1-4]$, the relation between the three terms was drastically changed as shown in Figure 2(b). For $\theta_{13}=10^{\circ}$, the $\mathrm{CP}$ interference term at $L / E=500 \mathrm{~km} / \mathrm{GeV}$ is much smaller than the dominant atmospheric term, whereas it has about the same amplitude as the dominant atmospheric term at $L / E=1500 \mathrm{~km} / \mathrm{GeV}$. From these considerations it is thus clear that the sensitivity to $\delta_{\mathrm{CP}}$ indeed is significantly higher at the second neutrino oscillation maximum than at the first.

The conceptual design of ESSnuSB [9] was made in 2012 and in view of the, at the time, newly measured high value of $\theta_{13}$, the detector was located at the second neutrino oscillation maximum. For an ESSnuSB proton energy of $2.0 \mathrm{GeV}$ the mean neutrino energy is about $360 \mathrm{MeV}$ and the distance of the second maximum from the $\nu_{\mu}$ source is of the order of $540 \mathrm{~km}$. Figure 3 shows, for this baseline, the number of electron neutrinos detected in two years, running with positive polarity in the horn, as a function of neutrino energy $E$ for four different values of $\delta_{\mathrm{CP}}: 0$ and $\pi$, which correspond to no CP violation, and $\pi / 2$ and $3 \pi / 2$, which correspond to maximum CP violation of opposite signs, respectively. The parameters used in the GLoBES calculation to obtain this plot are $\Delta m_{21}^{2}=7.5 \cdot 10^{-5} \mathrm{eV}^{2}, \Delta m_{31}^{2}=2.47 \cdot 10^{-3} \mathrm{eV}^{2}, \theta_{12}=0.58$, 


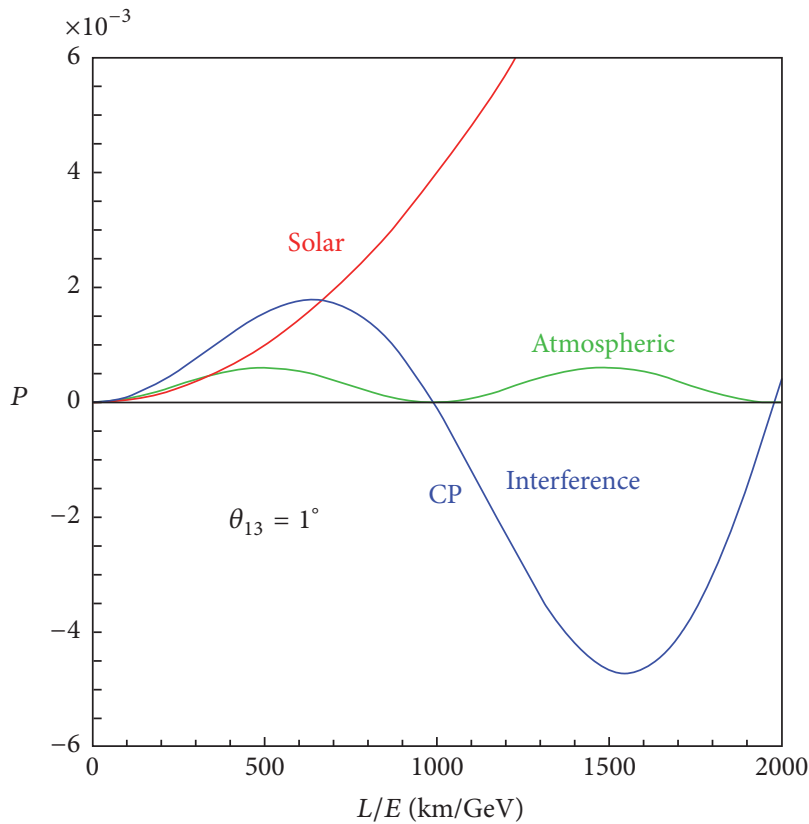

(a)

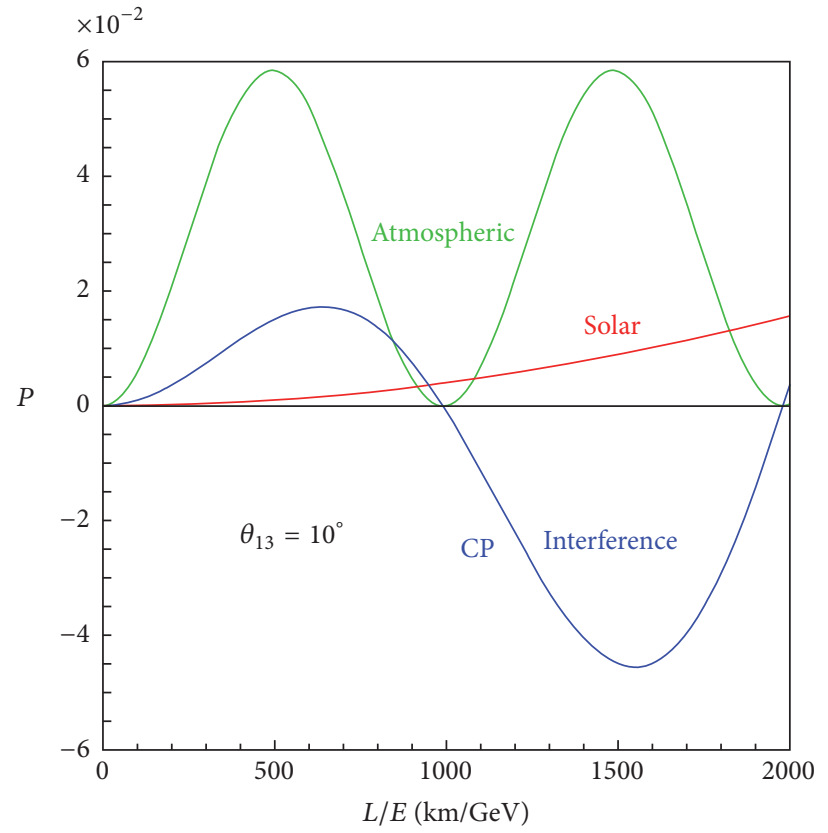

(b)

Figure 2: Plots showing, for two different values of the neutrino mixing angle $\theta_{13}=1^{\circ}$ (a) and $\theta_{13}=10^{\circ}$ (b), the three terms in the expression for the $v_{\mu} \rightarrow v_{e}$ oscillation probability as a function of the ratio between the accelerator-to-detector distance $L$ (the baseline) and the neutrino energy $E$ [19]. The $\mathrm{CP}$ violating term is calculated without the factor $\cos \left(\delta_{\mathrm{CP}}-\Delta_{31} L / 2\right)$; that is, what is shown is the maximum value this term could take on.

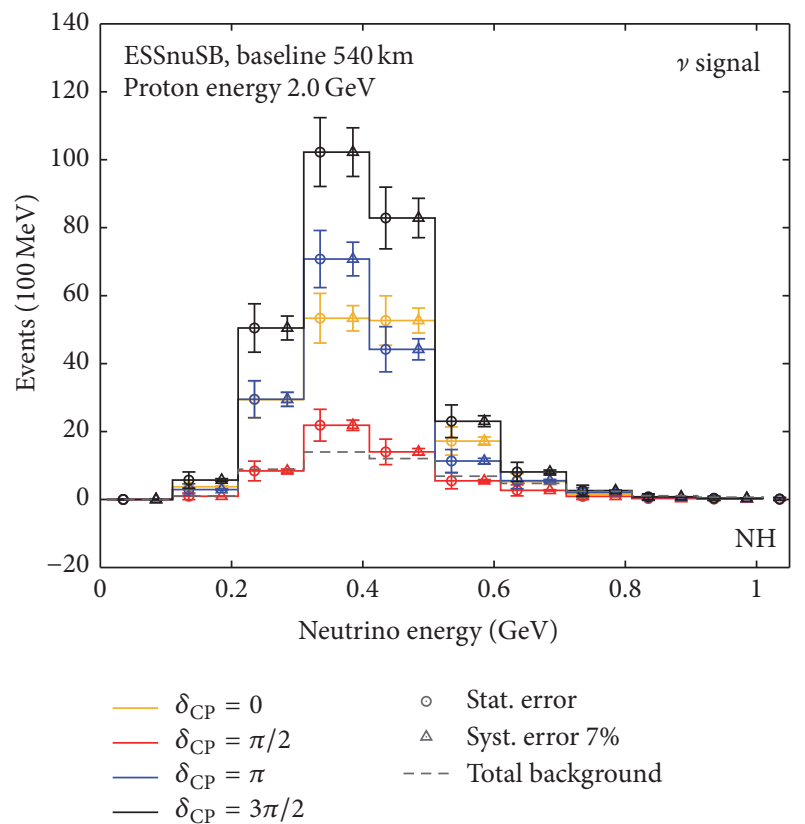

Figure 3: Histograms showing, for a baseline of $540 \mathrm{~km}$ and a proton energy of $2.0 \mathrm{GeV}$, the energy distribution for the electron neutrinos detected during 2 years of data taking for three different values of $\delta_{\mathrm{CP}}$.

$\theta_{13}=0.15$, and $\theta_{23}=0.70$. These parameters are included assuming prior knowledge with an accuracy of $3 \%$ for $\theta_{12}$, 0.02 for $\sin ^{2} 2 \theta_{23}, 0.005$ for $\sin ^{2} 2 \theta_{13}, 4 \%$ for $\Delta m_{31}^{2}$, and $3 \%$ for $\Delta m_{21}^{2}$ at $1 \sigma$ level. The neutrino mass hierarchy is assumed to be unknown. The same parameters have been used for all results of ESSnuSB calculations shown in this paper.

Figure 4 shows the same type of comparison, this time between DUNE and Hyper-K, designed to measure at the first maximum, and ESSnuSB, designed to measure at the second maximum. A comparison of the left and right panels shows that almost all of the events in the Hyper- $\mathrm{K}$ and DUNE experiments are located within the region of the first oscillation maximum whereas for ESSnuSB almost all of the events are located within the region of the second maximum. In particular, there is no increase in the signal count rate visible for Hyper-K or DUNE in the energy region of the second maximum. The ratio between the numbers of electron neutrinos with $\delta_{\mathrm{CP}}=3 \pi / 2$ and with $\delta_{\mathrm{CP}}=\pi / 2$ can be seen to be about 1.5 for DUNE, 1.6 for Hyper-K, and as high as about 4.8 for ESSnuSB, implying an about three times higher sensitivity to the value of $\delta_{\mathrm{CP}}$ as compared to the other two experiments.

The four bins in Figure 5 show the total number of events detected at the second maximum for neutrinos and antineutrinos and for a proton energy of $2 \mathrm{GeV}$ and $2.5 \mathrm{GeV}$, respectively. The data collection time is 2 years with neutrinos and 8 years with antineutrinos in order to detect (very) approximately equal numbers of electron neutrinos and antineutrinos. For a proton energy of $2.5 \mathrm{GeV}$ the total number of events can be seen to be somewhat higher than for a $2.0 \mathrm{GeV}$ proton energy, indicating that the energy originally planned for the ESS project of $2.5 \mathrm{GeV}$ would be more favorable for ESSnuSB than the recently downscoped value of $2.0 \mathrm{GeV}$. 

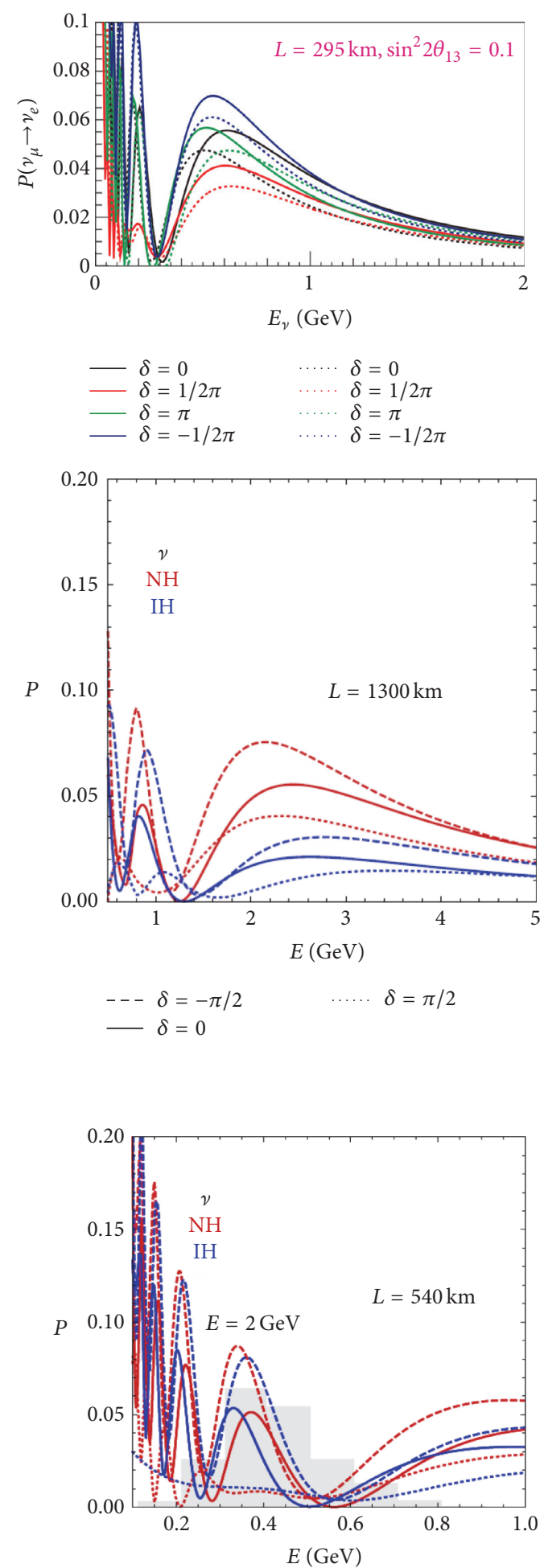

$$
\begin{aligned}
--\delta & =-\pi / 2 \quad \cdots \cdots \delta=\pi / 2 \\
-\delta & =0
\end{aligned}
$$

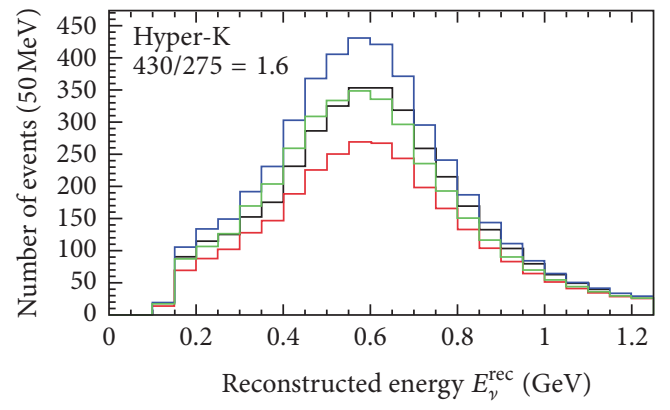

$-\delta=0 \quad-\delta=-90$

$\delta=90 \quad-\delta=180$
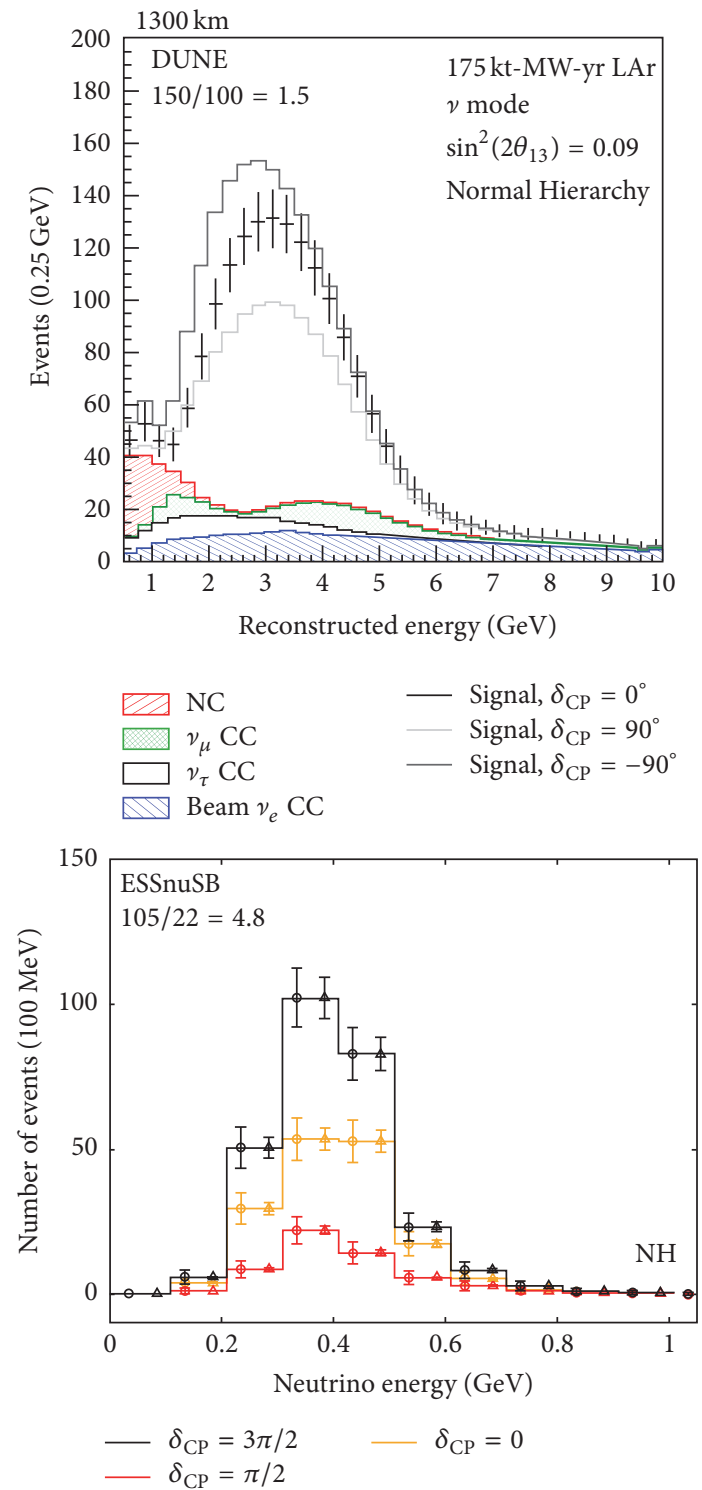

FIGURE 4: The histograms in the panels in the right hand part of the figure show, for the three different neutrino beam projects Hyper-K [8], DUNE [20, 21], and ESSnuSB, the number of detected electron neutrinos as a function of neutrino energy $E$ for three different values of $\delta_{\mathrm{CP}}$ : 0 , which corresponds to no CP violation, and $\pi / 2$ and $3 \pi / 2$, which correspond to maximum CP violation of opposite signs, respectively. The left panels show the calculated probability for the electron neutrino oscillation for the same three $\delta_{\mathrm{CP}}$ values and for Normal Hierarchy $(\mathrm{NH}$, red) and Inverted Hierarchy (IH, blue) $[8,21]$. The shaded histogram in the left ESSnuSB panel shows the profile of the unoscillated (muon) neutrino energy distribution. The ratio between the numbers of electron neutrinos with $\delta_{\mathrm{CP}}=3 \pi / 2$ and with $\pi / 2$ is 4.8 for ESSnuSB and 1.6 and 1.5, respectively, for Hyper-K and LBNE/DUNE, as noted under the experiment acronym in each histogram graph. 


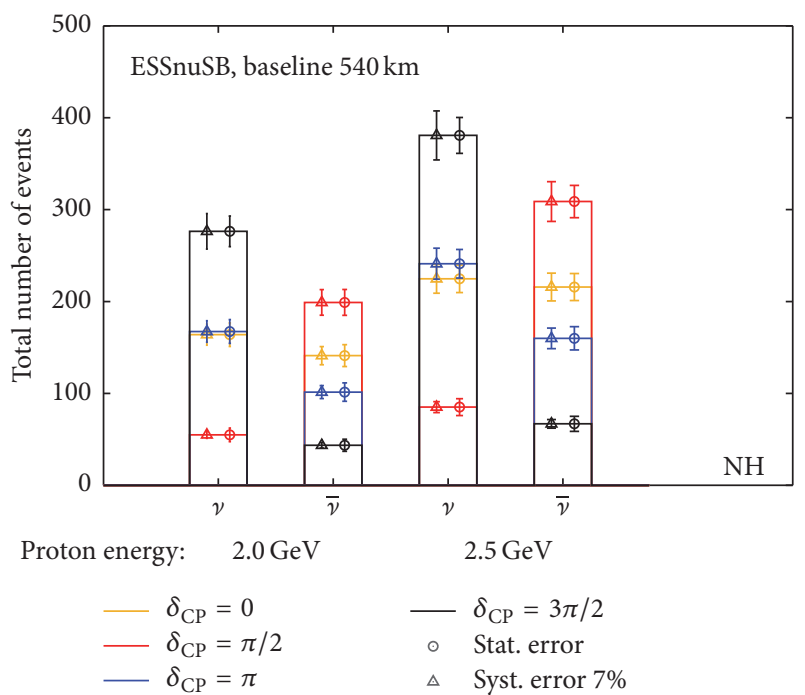

FIGURE 5: The bins show the total numbers of electron neutrinos and antineutrinos, respectively, detected in ESSnuSB after 2 years of data taking with a neutrino beam and 8 years with an antineutrino beam and for a proton energy of $2.0 \mathrm{GeV}$ and for $2.5 \mathrm{GeV}$, respectively, with the detector placed at the second maximum.

The T2K experiment [22] has, after 4 years of operation and data analysis, managed to reduce its systematic uncertainties for the electron (muon) neutrino signal to a level of $6.3 \%$ (7.4\%). Figure 3 shows, for each $100 \mathrm{MeV}$ bin, the statistical errors as well as a $7 \%$ systematic error. The statistical error is seen to be larger than the systematic one. However, as shown by the leftmost bar in Figure 5, which represents the total numbers of events in the histograms in Figure 3, for the total number of events the statistical and systematic errors are in balance. This raises the question of how much information is contained in the relative shape of the histograms in Figure 3, as this information is not taken into account when considering only the total number of events. The answer to this question may be deduced from the four different histograms in Figure 6, which have been obtained by dividing the four histograms of Figure 3 by their respective total numbers of events. There is some difference between the curves in Figure 6 but these differences are comparable in magnitude to the statistical errors.

Described so far is what could be done with ESSnuSB neutrino beam data collected during 2 years. However, a major goal is to compare the neutrino and antineutrino beam data shown in Figure 5, which provides additional and very sensitive information on $\delta_{\mathrm{CP}}$. Figure 7 shows for ESSnuSB the normalized difference between the total numbers of electron neutrinos, collected during 2 years, and of electron antineutrinos, collected during 8 years $\left(N_{v_{e}}-N_{\bar{v}_{e}}\right) /\left(N_{v_{e}}+\right.$ $N_{\bar{v}_{e}}$ ) for a proton energy of $2.0 \mathrm{GeV}$ and $2.5 \mathrm{GeV}$, respectively, at the first maximum (a) and the second maximum (b). The normalization takes into account the difference in the production as well as the detection cross sections between the neutrinos and the antineutrinos. The variation of the asymmetry with $\delta_{\mathrm{CP}}$ can be seen to be significantly bigger at the second maximum as compared to the first maximum and

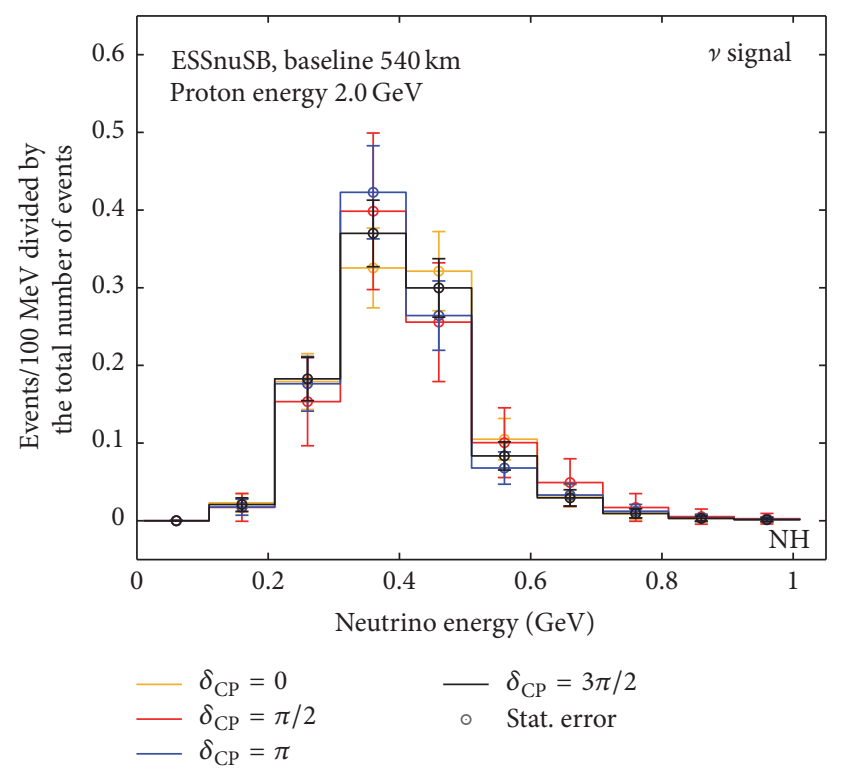

Figure 6: The 4 different histograms in this figure have been obtained by dividing each of the four different histograms in Figure 3 by their respective total numbers of events, so that the difference in relative shape between the histograms can be seen.

the statistical and systematic errors are well balanced. Other investigations of the sensitivity of the neutrino-antineutrino asymmetry to the value of $\delta_{\mathrm{CP}}$ have shown that the probability $P\left(\bar{v}_{\mu} \rightarrow \bar{v}_{e}\right)$ varies with $\delta_{\mathrm{CP}}$ between $1 / 2$ and 2 times the probability $P\left(\nu_{\mu} \rightarrow v_{e}\right)$ at the first maximum and between 1/7 and 7 times the probability $P\left(v_{\mu} \rightarrow v_{e}\right)$ at the second maximum [23].

By fitting simulated $[24,25]$ ESSnuSB data collected during 2 years with a neutrino beam and 8 years with an antineutrino beam one may calculate the fraction of the total range of possible values for $\delta_{\mathrm{CP}}$ for which $\mathrm{CP}$ violation can be discovered with $5 \sigma$ and $3 \sigma$ significance level, respectively. In Figure 8 the result of such global calculations is plotted for different distances between the accelerator and the detector $L$ from $100 \mathrm{~km}$ to $1000 \mathrm{~km}$ (horizontal axis), for the three different proton beam energies 2.0, 2.5, and $3.0 \mathrm{GeV}$ (blue, green, and red lines, resp.), and with the mixing angle $\theta_{13}=8.73^{\circ}$. From these curves one may see that the highest potential for discovery is at the second maximum. One can also see that the potential is somewhat higher for a proton beam energy of $2.5 \mathrm{GeV}$ (and $3.0 \mathrm{GeV}$ ) as compared to $2.0 \mathrm{GeV}$. The systematic errors used to produce these plots are those shown in the left column (SB Def.) of Table 3 [18].

With the exceptionally high power of the ESS linac it is possible to profit from the significantly higher sensitivity to $\delta_{\mathrm{CP}}$ at the second maximum, rendering ESSnuSB about three times less sensitive to systematic errors as compared to experiments measuring at the first maximum.

Figure 9 shows, for ESSnuSB using a $540 \mathrm{~km}$ baseline and a proton energy of $2.0 \mathrm{GeV}$ ((a) current ESS design) and $2.5 \mathrm{GeV}$ ((b) originally planned linac energy for which upgrade space is available in the linac tunnel), respectively, the dependence of the fraction of values of $\delta_{\mathrm{CP}}$ for which 


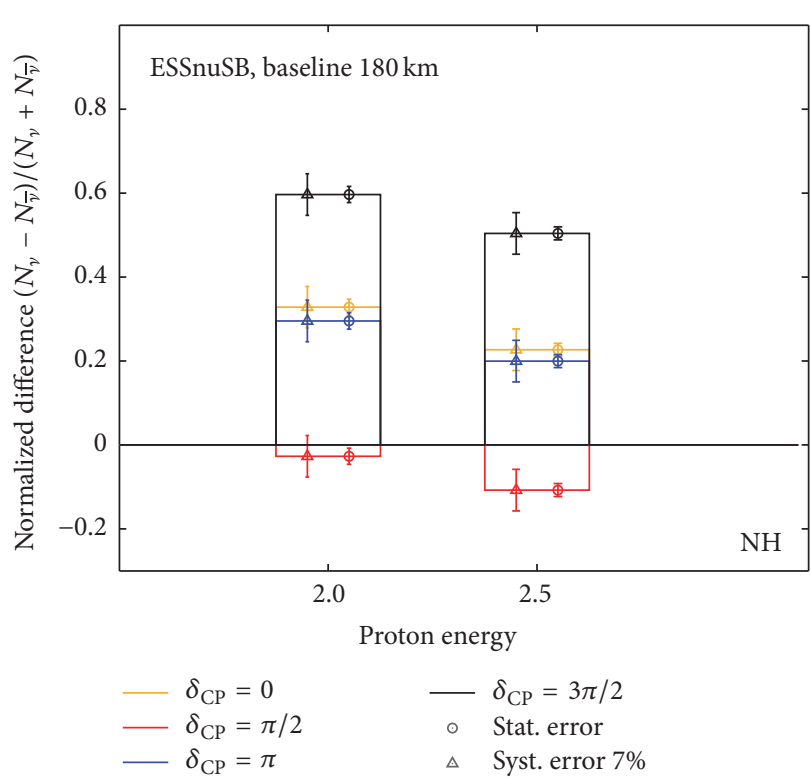

(a)

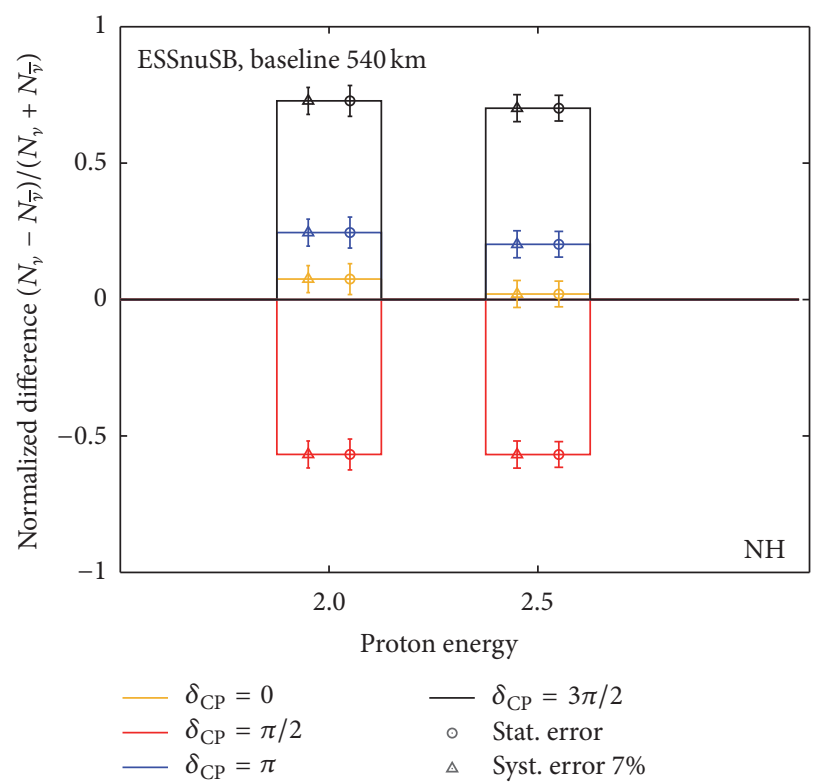

(b)

FIGURE 7: The towers show the normalized difference between the total numbers of electron neutrinos and of electron antineutrinos $\left(N_{v_{e}}-\right.$ $\left.N_{\bar{v}_{e}}\right) /\left(N_{v_{e}}+N_{\bar{v}_{e}}\right)$ for a proton energy of $2.0 \mathrm{GeV}$ and $2.5 \mathrm{GeV}$, respectively, at the first maximum (180 km (a)) and the second maximum (540 km (b)).

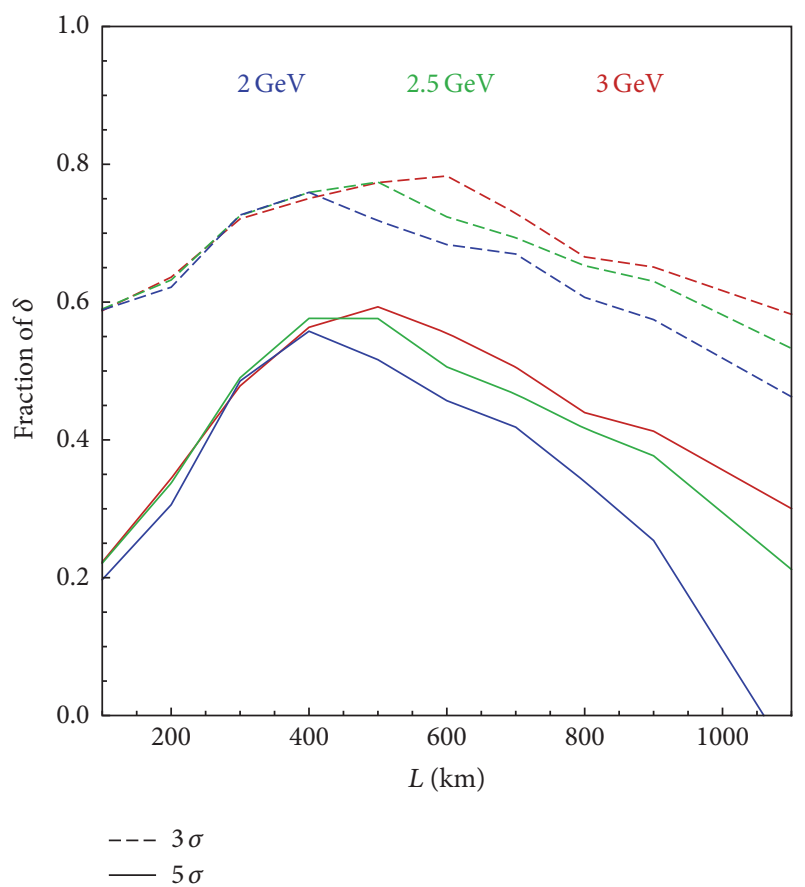

FIGURE 8: The curves show the fraction of the total range of possible values for $\delta_{\mathrm{CP}}$ for which $\mathrm{CP}$ violation can be discovered with $5 \sigma$ and $3 \sigma$ significance level, respectively, as a function of the distance $L$ between the accelerator and the detector (the baseline), with $L$ ranging from $100 \mathrm{~km}$ to $1000 \mathrm{~km}$ (horizontal axis) for three different proton beam energies 2.0,2.5, and $3.0 \mathrm{GeV}$ (blue, green, and red curves) and for a value of the mixing angle $\theta_{13}=8.73^{\circ}$. The systematic errors used to produce these plots are those listed in the left column (SB Def.) of Table 3.
TABLE 3: The different sources of uncertainty that contribute to the total systematic uncertainty in the determination of the $\mathrm{CP}$ violating angle $\delta_{\mathrm{CP}}$. This table is an extraction of information from a table used in [18]. Here, only the uncertainties for the Super Beam cases "SB Def." (for default) are used to obtain the results shown in Figures 8 and 10 and "SB Opt." (for optimistic) to obtain the results shown in Figure 9.

\begin{tabular}{lcc}
\hline Error source & SB Def. & SB Opt. \\
\hline Fiducial volume ND & $0.5 \%$ & $0.2 \%$ \\
Fiducial volume FD & $2.5 \%$ & $1.0 \%$ \\
Flux error signal $\nu$ & $7.5 \%$ & $5 \%$ \\
Flux error background $\nu$ & $15 \%$ & $10 \%$ \\
Flux error signal $\bar{\nu}$ & $15 \%$ & $10 \%$ \\
Flux error background $\bar{\nu}$ & $30 \%$ & $20 \%$ \\
Background uncertainty & $7.5 \%$ & $5 \%$ \\
\hline Cross sections $\times$ eff. QE & $15 \%$ & $10 \%$ \\
Cross sections $\times$ eff. RES & $15 \%$ & $10 \%$ \\
Cross sections $\times$ eff. DIS & $7.5 \%$ & $5 \%$ \\
Effec. ratio $\nu_{e} / \nu_{\mu}$ QE & $11 \%$ & $3.5 \%$ \\
Effec. ratio $\nu_{e} / \nu_{\mu}$ RES & $5.4 \%$ & $2.7 \%$ \\
Effec. ratio $\nu_{e} / \nu_{\mu}$ DIS & $5.1 \%$ & $2.5 \%$ \\
\hline Matter density & $2 \%$ & $1 \%$ \\
\hline
\end{tabular}

a $\mathrm{CP}$ violation discovery at $5 \sigma$ and $3 \sigma$, respectively, can be made as a function of event statistics, or as it is called in this figure "exposure." The "nominal exposure" corresponds to 10 years of data taking: 2 years with a neutrino beam and 8 years with an antineutrino beam. The systematic errors that have been used are shown in the right column (SB Opt.) of 


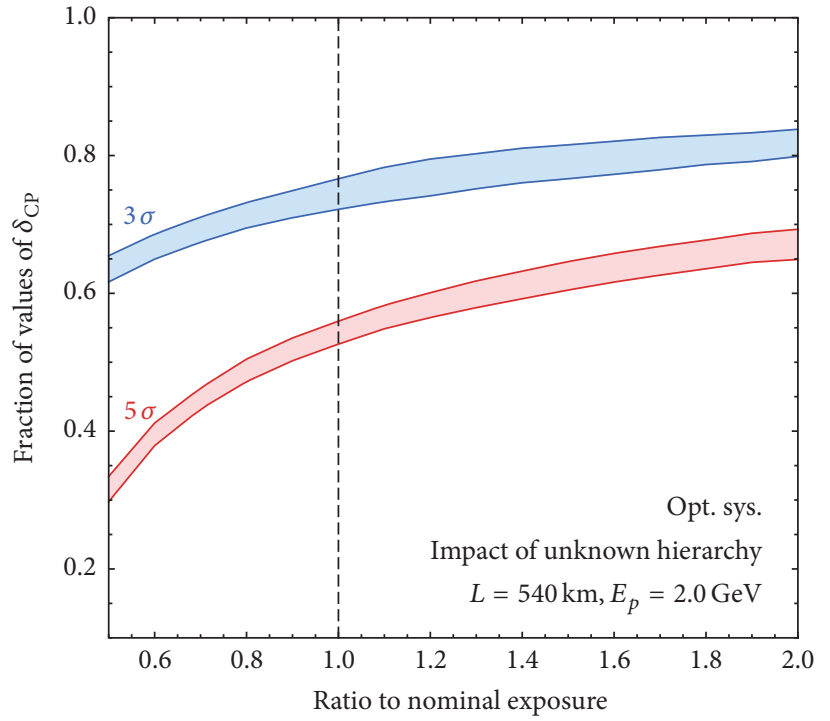

(a)

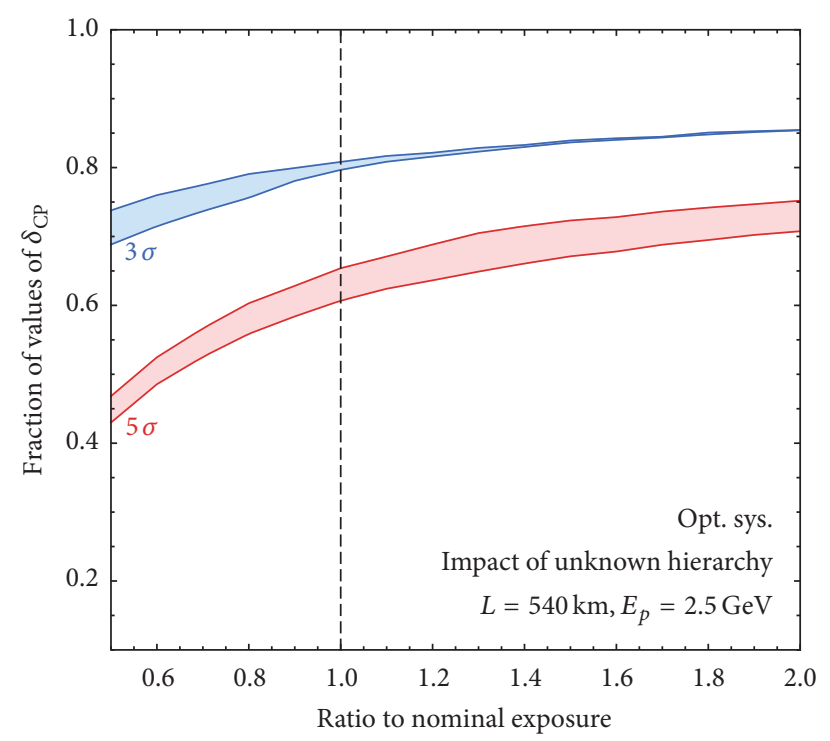

(b)

FIGURE 9: Curves showing the dependence of the fraction of values of $\delta_{\mathrm{CP}}$ for which a CP violation discovery at $5 \sigma$ and $3 \sigma$, respectively, can be made as a function of event statistics, or exposure, where the nominal exposure corresponds to 10 years of data taking. (a) is for a proton energy of $2.0 \mathrm{GeV}$ (current ESS design) and (b) for $2.5 \mathrm{GeV}$. The upper boundaries of the colored bands were obtained assuming the hierarchy to be known $(\mathrm{NH})$ and the lower boundaries assuming the hierarchy to be unknown; that is, it is marginalized over. The systematic errors used to produce these plots are those shown in the right column (SB Opt.) of Table 3 [18].

Table 3. The upper boundary of the colored bands assumes the neutrino hierarchy to be known and the lower boundary to be unknown. For the nominal exposure, assuming the neutrino hierarchy to be known, $\mathrm{CP}$ violation can be discovered at $5 \sigma$ in about $56 \%$ and $65 \%$ of the total range of possible values, for a $2.0 \mathrm{GeV}$ and a $2.5 \mathrm{GeV}$ linac proton energy, respectively. Doubling the exposure to 4 years with a neutrino beam and 16 years with an antineutrino beam will lead to an increase of the fraction of the total range of possible $\delta_{\mathrm{CP}}$ covered to $69 \%$ and $71 \%$, respectively. The comparatively small difference in this fraction when doubling the exposure, assuming the hierarchy to be known and unknown, illustrates the comparatively weak dependence on this parameter at the low neutrino energies of ESSnuSB.

In order for DUNE to reach a similar performance, a $2 \%$ systematic error in the signal normalization for $v_{e}$ is required [7]. For Hyper- $\mathrm{K}$ the corresponding requirement is a total systematic error of $4 \%$ in the energy region of maximal flux $0.55-0.65 \mathrm{GeV}$ [8]. The current systematic error in the $\mathrm{T} 2 \mathrm{~K}$ measurement of $\nu_{e}$ appearance is $6.3 \%$ [22]. It is quite clear that the systematic errors shown in the "SB Opt." column of Table 3 , in which the neutrino flux systematic error is $5 \%$ and the neutrino cross section systematic error is $10 \%$ (at the low neutrino energies of ESSnuSB the QE cross section dominates), are considerably more readily obtainable as compared to the total systematic error levels of $2 \%$ and $4 \%$ required for DUNE and Hyper-K, respectively.

Conversely, assuming the systematic errors to be on the same level for all three experiments as for ESSnuSB, the potential for CP discovery is higher for ESSnuSB due to the approximately three times higher signal at the second maximum. This was confirmed already in 2013 by global calculations, made by the theory group at the Snowmass Study in the USA [26], in which the expected performances of the different proposals for international neutrino beam projects were compared, using the same systematic errors for all experiments, in this case the systematic errors shown in the left column (SB Def.) of Table 3. Figure 10(a) shows the $1 \sigma$ error in the determination of $\delta_{\mathrm{CP}}$ (horizontal axis) as a function of the covered fraction of $\delta_{\mathrm{CP}}$ (vertical axis). One can see that, among the accelerator based projects shown, the resolution attainable with ESSnuSB is surpassed only by the Neutrino Factory project (IDS-NF). Figure 10(b) shows with which level of significance, in terms of number of standard deviations $\sigma, \mathrm{CP}$ violation can be discovered (vertical axis) versus the fraction of the total range of possible values for $\delta_{\mathrm{CP}}$ for which $\mathrm{CP}$ violation (horizontal axis) can be discovered. From these plots ESSnuSB can be seen to have the widest discovery coverage of the $\delta_{\mathrm{CP}}$ range among the Super Beam experiments investigated. Admittedly, the systematic errors chosen for these studies made in 2013 are quite conservative; for example, the error for the $v_{e}$ signal is $7.5 \%$, but this is to our knowledge the only comparison made under the assumption of equal systematic errors for all experiments. There is in principle no reason why there should be a significant difference in the systematic errors between the future long baseline experiments, at least not between Hyper- $\mathrm{K}$ and ESSnuSB which are planned to have similar neutrino beam energies and neutrino detectors. The ca. three times higher CP signal in ESSnuSB does however represent a significant difference. 


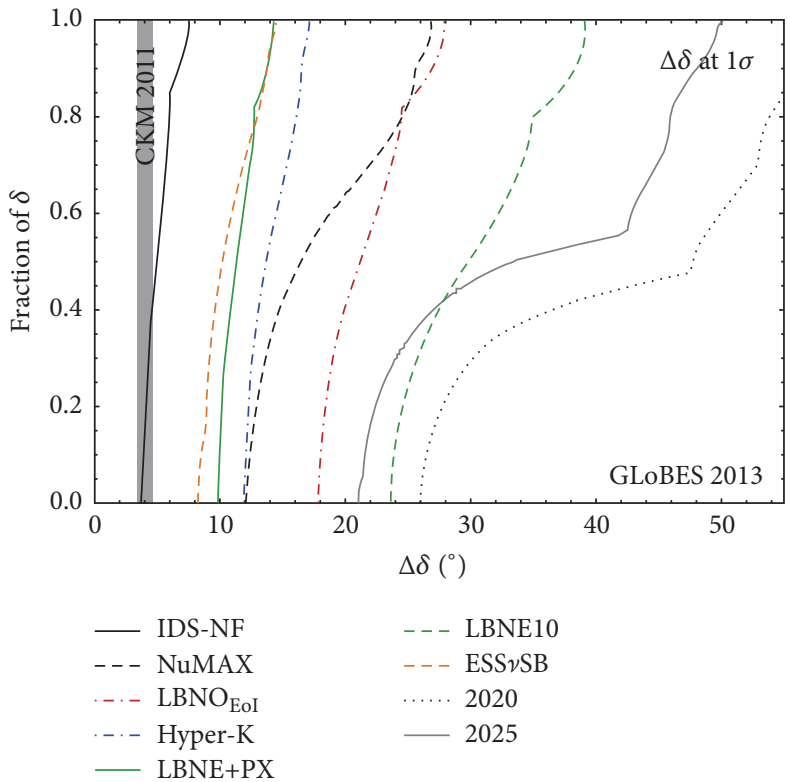

(a)

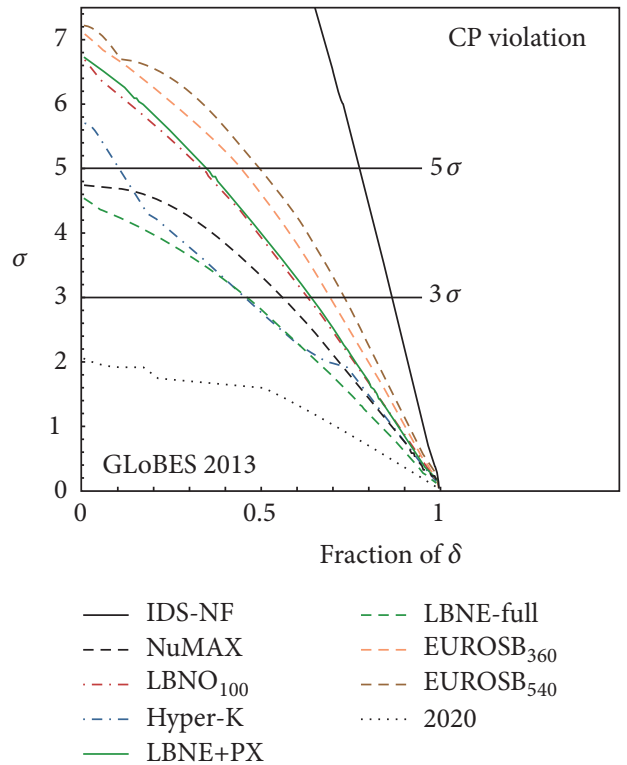

(b)

Figure 10: In (a) the $1 \sigma$ error in the determination of $\delta_{\mathrm{CP}}$ is shown (horizontal axis) as a function of the $\delta_{\mathrm{CP}}$ fraction for which this accuracy can be reached (vertical axis) [26]. (b) shows with what level of significance in terms of number of standard deviations $\sigma$ that leptonic CP violation can be discovered (vertical axis) versus the fraction of the total range of possible values for $\delta_{\mathrm{CP}}$ (horizontal axis) [9]. The systematic errors used to produce these plots are those shown in the left column (SB Def.) of Table 3.

\section{The Required Upgrades and Additions to the ESS Accelerator Facility}

The $2.86 \mathrm{~ms}$ long proton pulses at ESS will be sent directly to the spallation target for neutron production. However, for neutrino production the requirement to have a few $\mu$ s short pulses on target makes it necessary to compress the proton pulses using an accumulator ring. The injection of many turns into this ring cannot be made efficiently if the injected beam consists of the same particles as the circulating beam. By accelerating $\mathrm{H}^{-}$ions in the linac, an efficient injection system can be designed based on the use of stripping foils or a laser beam, thus producing a circulating proton beam in the accumulator. From this follows the fact that the linac must be able to accelerate interleaved pulses of protons and $\mathrm{H}^{-}$. In particular the linac magnets must be designed such that it will be possible to switch between two different optics settings for the two different linac beams.

At the US Spallation Neutron Source (SNS), at Oakridge National Laboratory [27], the linac also accelerates $\mathrm{H}^{-}$pulses that are compressed through multiturn injection into an accumulator, to get shorter pulses for their neutron production target. The beam power at SNS is about $1.5 \mathrm{MW}$ on target [27] and foil stripping is used. Due to the similar context and requirements, we have used the SNS accumulator as a model for our first studies of the ESS accumulator.

A second ion source, for the production of $\mathrm{H}^{-}$, will be needed at ESS, as well as additional equipment in an $\mathrm{H}^{-}$ injector line for beam transport up to the point where the $\mathrm{H}^{-}$ beam goes into ESS linac. The optimal place for merging the two lines remains to be identified on the basis of the beam transport efficiency from the source.
$\mathrm{H}^{-}$ions in the linac beam will lose electrons due to phenomena such as collisions with residual gas, with blackbody photons, and by intrabeam stripping. This will lead to some additional beam loss which needs to be minimized. Lorentz stripping in the magnetic fields can be reduced by careful choice of the optics for the $\mathrm{H}^{-}$beam. Considering the experience of SNS it has been concluded that the losses in the ESS linac for the $\mathrm{H}^{+}$case would be around $0.1 \mathrm{~W} / \mathrm{m}$. To avoid complicating the service work for the linac, the total losses for both beams in the linac, both $\mathrm{H}^{-}$and $\mathrm{H}^{+}$, should be maximum $1.0 \mathrm{~W} / \mathrm{m}$ and this total limit will have to be guaranteed by the design of the transport of the $\mathrm{H}^{-}$beam and by the collimation system.

The $\mathrm{H}^{-}$linac pulse will be chopped in the medium energy beam transport line $(3.6 \mathrm{MeV})$ to have regular gaps in the pulse such that when the beam is fully injected in the accumulator, there is a gap in the circulating beam. This is needed in order to avoid beam loss during the radio frequency (rf) beam capture in the accumulator and for the extraction of the beam to the production target. These gaps are seen by the accelerating cavities in the linac. The resulting higher order resonance modes (HOMs) are not expected to be an issue; however, this will be verified by calculations. The fact that there is no beam in the gaps represents a loss of about $10 \%$ of the particles on the production target.

The baseline energy of the linac beam is $2.0 \mathrm{GeV}$. Contingency space is available in the linac tunnel for upgrade to higher energies. As mentioned in Section 2, higher energies are beneficial for the physics reach of the ESSnuSB facility. The extraction of the H-beam will be made in the contingency region of the linac tunnel (see Figure 1), at the point where $2.5 \mathrm{GeV}$ can be reached by adding more accelerating modules, 


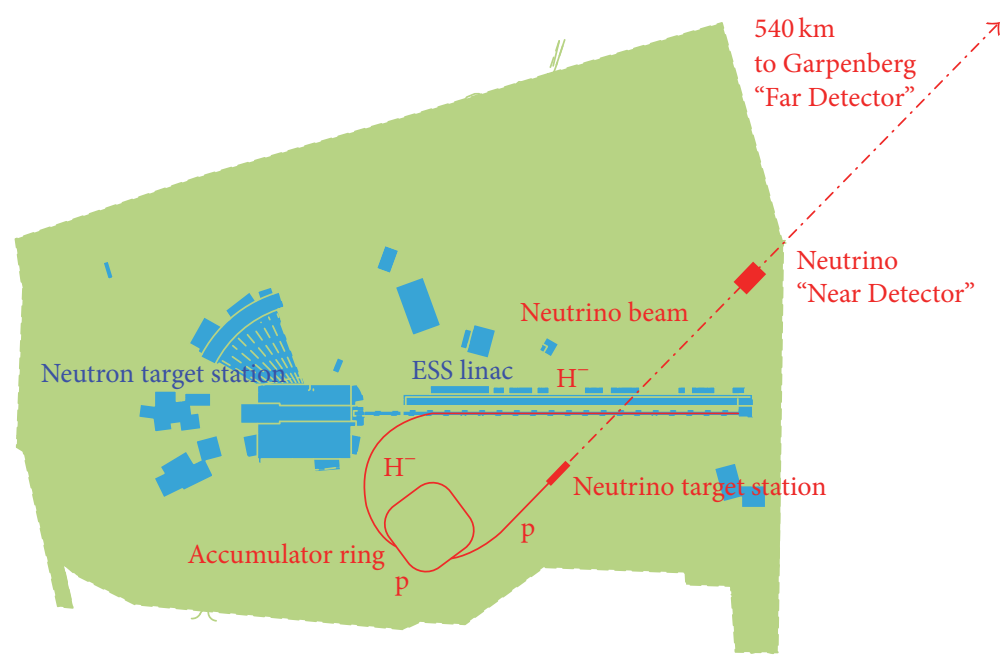

FIGURE 11: The proposed layout on the ESS site (courtesy of K. Hedin, ESS). The $\mathrm{H}^{-}$beam in the linac, the accumulator and the transfer lines, the neutrino target station, and the neutrino beam are shown in red.

such that it will be possible to profit from a future linac upgrade to this energy. Due to space limitations around the neutron target hall, the transfer line from the linac to the accumulator cannot be designed for a beam energy higher than $2.5 \mathrm{GeV}$. This limitation is due to the requirement that the radius of the $\mathrm{H}^{-}$beam transfer lines must be large enough so that the induced radioactivity by Lorentz stripping can be kept within allowed limits. With a $66 \%$ dipole filling factor in the transfer line and $2.5 \mathrm{GeV}$ beam energy, the radius must

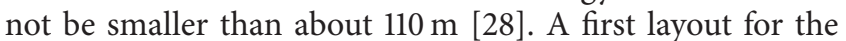
accumulator, the target, the transfer lines, and the neutrino beam direction is shown in Figure 11. The target station would need to be located at a depth of $25 \mathrm{~m}$ (see Section 4), and the neutrino beam will therefore pass below the linac.

There are several additional important items in the inventory of upgrades that need to be made during the linac construction phase for the future additional $5 \mathrm{MW} \mathrm{H}^{-}$ beam, such as the extra infrastructure and space that will be required for additional cabling and electrical equipment, for extra power transformers, for the additional $\mathrm{H}^{-}$source, and for the beam equipment of the $\mathrm{H}^{-}$beam transport at low energy. The water cooling plant, the cryogenic liquids plant, the cooling channels in the radio frequency power sources, and the accelerating cavities need to be designed for increased dynamic heat load [15]. It has to be possible to adjust the strengths of the linac focusing and steering magnets with the linac pulsing frequency. It is essential that these comparatively modest preparatory modifications be made during the linac build-up phase 2017-2023 in order to reduce the cost and time required for the subsequent linac upgrade and, in particular, to reduce the disruption in the linac operation for neutron production to an acceptable level.

3.1. The Accumulator. To fill the accumulator with as many as $1.1 \cdot 10^{15}$ protons is a challenging task. This number could be reduced by splitting the $2.86 \mathrm{~ms}$ linac pulse in shorter and more frequent linac pulses of the same current, still maintaining the $5 \mathrm{MW}$ total power for the neutrinos [29].
TABLE 4: Summary of lattice parameters for the accumulator.

\begin{tabular}{lc}
\hline Parameter & Value \\
\hline Circumference & $376 \mathrm{~m}$ \\
Number of dipoles & 64 \\
Number of quadrupoles & 84 \\
Injection region & $12.5 \mathrm{~m}$ \\
Revolution time & $1.32 \mu \mathrm{s}$ \\
\hline
\end{tabular}

The first case under study is to reduce the maximum intensity in the ring to $1 / 4$. One $2.86 \mathrm{~ms}$ long pulse would be sent to the neutron target and 4 pulses, $0.72 \mathrm{~ms}$ long, to the accumulator, respectively. This can be achieved by increasing the pulsing rate of the linac from $28 \mathrm{~Hz}$ to $70 \mathrm{~Hz}$; see the lower part of Figure 12. The neutron pulse frequency would still be $14 \mathrm{~Hz}$. Alternatively, the $2.86 \mathrm{~ms}$ pulse could be injected sequentially into four stacked accumulator rings using a switchyard at injection as proposed in [9]. Also in this configuration the intensity in each ring would be $1 / 4$ of the total intensity.

Studies are ongoing to evaluate effects of the high intensities using a first design of an accumulator with $376 \mathrm{~m}$ circumference and $1.32 \mu$ s revolution time. The main parameters are shown in Table 4 and the lattice layout in Figure 13. The studies will indicate the intensity limit of the ring and possible improvements of the design. The aim is to have as few rings and as low linac pulsing frequency as possible. Having only one ring needs careful design of the stripping foil, which has to accept higher pulse rates than in the case of four stacked rings. The option with several stacked rings is subject to Lorentz stripping in the injection switchyard that distributes the linac beam into the four rings. Beam loss from this beam switching would have to be evaluated [30].

Increasing the number of pulses leads to increased power consumption in the cavities because of the power losses when ramping up and down the radio frequency field strength in the cavities for each pulse. $5 \mathrm{MW}$ beam power needs 13.3 MW 

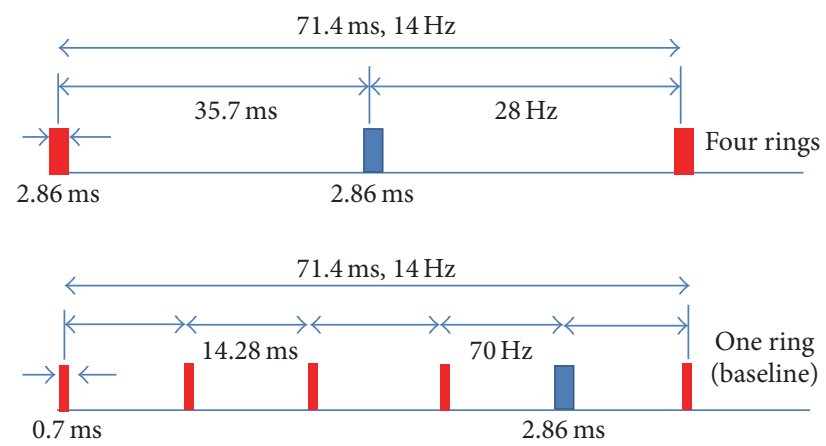

Figure 12: Pulse distribution for the case with four stacked accumulators and for the one-ring options: the upper part of the figure shows the $28 \mathrm{~Hz}$ pulsing of the linac with one proton (blue) and one $\mathrm{H}^{-}$(red) pulse interleaved and the lower part shows the case of four $0.72 \mathrm{~ms}$ long pulses of $\mathrm{H}^{-}$for neutrinos which are followed by one $2.86 \mathrm{~ms}$ proton pulse for the neutrino spallation target. The upper case needs a switching system and gaps in the $\mathrm{H}^{-}$pulse to distribute the particles in the four stacked rings.

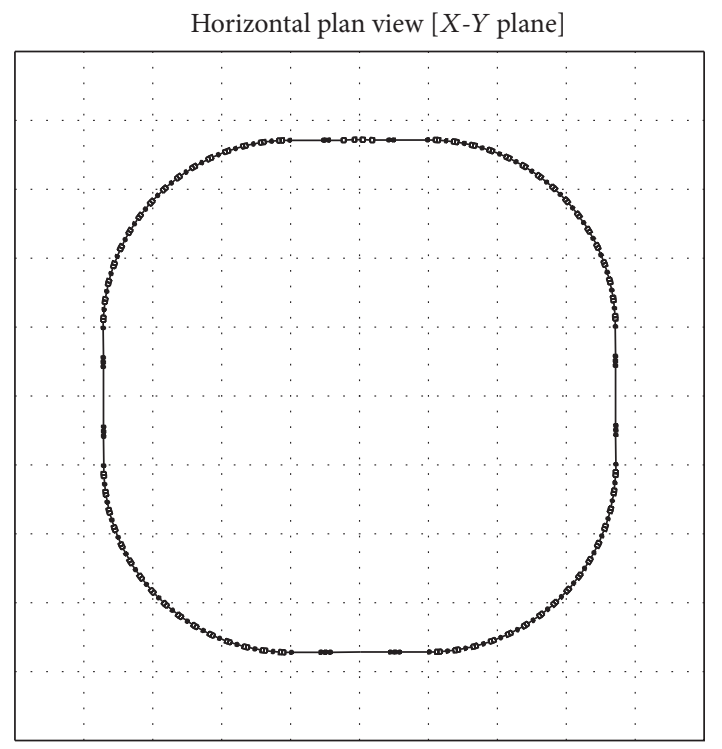

Grid size $15.0000(\mathrm{~m})$

FIgURE 13: The layout of the lattice for the ESS accumulator.

wall plug power for 14 pulses of $2.86 \mathrm{~ms}$ per second. In the case of one accumulator ring and $70 \mathrm{~Hz}$ pulse frequency (see Figure 12) 13.3 MW + 17.0 MW wall plug power will be needed. The cost of the additional 3.7 MW, essentially being the overhead to fill and empty the cavities, will have to be weighed against the cost of the construction, the power consumption, the operation of three additional accumulators, and the impact on the target pulsing. Ongoing testing of the cavities at the FREIA Laboratory [31] at Uppsala University and future operational experience of the ESS linac will show how many pulses per second can be safely produced. The choice of linac pulsing has impact also on the powering and pulsing of the target focusing system. From the EUROnu studies we have a design of a power supply that can be pulsed at $56 \mathrm{~Hz} .70 \mathrm{~Hz}$ needs development or an additional power supply.

3.1.1. Lattice. To store $2.7 \cdot 10^{14}$ protons in the accumulator requires a well chosen magnetic field structure (the lattice). The circumference and the beam pipe aperture are critical; the cost of investment and operation of the accumulator will critically depend on its design. The design concept of the accumulator ring at the SNS [27], in which the magnet fields in the original $1 \mathrm{GeV}$ lattice are rather moderate, has been used as a starting point for the design of the ESSnuSB accumulator. The lattice is a square lattice with four long straight sections for $\mathrm{rf}$, instrumentation, injection, and extraction; see Figure 13. It is possible to adopt the SNS $30 \mathrm{~m}$ injection straight section unchanged for $2.0 \mathrm{GeV}$. However, in view of the large apertures required, the bending fields in the arcs are kept as conservative as in the SNS; hence the arc lengths have tentatively been doubled considering that collective effects are more important at lower energies and that this would be conservative for $2.5 \mathrm{GeV}$. The circumference is increased from 248 to $376 \mathrm{~m}$, which reduces the number of injection turns per fill, which is an advantage for the foil temperature.

Different lattice types will be designed to study space charge effects. The first simulations have been made using the SNS FODO lattice [29]. A FODO lattice is flexible and robust and gives a compact beam size for high energy machines with, however, a relatively large variation around the ring in the transverse beam size [32]. Doublet lattices give more space in the lattice and are optimal for the focusing of highly nonspherical beams (mini- $\beta$ in electron colliders) and can lead to large variations in the transverse beam size. Triplets give a smooth variation of the beam size and, in particular, small variations of the ratio between the two transverse sizes (e.g., small $\beta$ in proton colliders) and an almost uniformly distributed space charge field.

3.1.2. Injection. To start the design procedure, a total final charge in the accumulator of $1 / 4$ of the original $1.1 \cdot 10^{15}$ protons has been assumed. This intensity gives negative space charge tune shifts of values less than 0.2 for an accumulator of $376 \mathrm{~m}$ having $100 \pi$ normalized (95\%) emittance fully injected beam, which is a value considered as conservative. The accumulator radio frequency system will keep the protons confined in an rf "bucket" and prevent the protons from filling the gap in the circulating beam in such a way that the beam can be extracted with low beam loss. As mentioned above, this gap will be generated in the linac medium energy section by chopping a part of the pulse corresponding to the gap duration. The gap duration will be determined by the extraction kicker rise time, which is between 50 and $100 \mathrm{~ns}$, the exact value depending on the design of the lattice and the angle of extraction.

Charge exchange injection by foil stripping is proposed as initial implementation, since this technology is well known. The SNS accumulator uses foil stripping in operation for a beam power of more than $1 \mathrm{MW}$. Laser stripping is envisaged at the SNS [33]. A future SNS laser stripping realization could ultimately be ported to the ESS accumulator with limited 


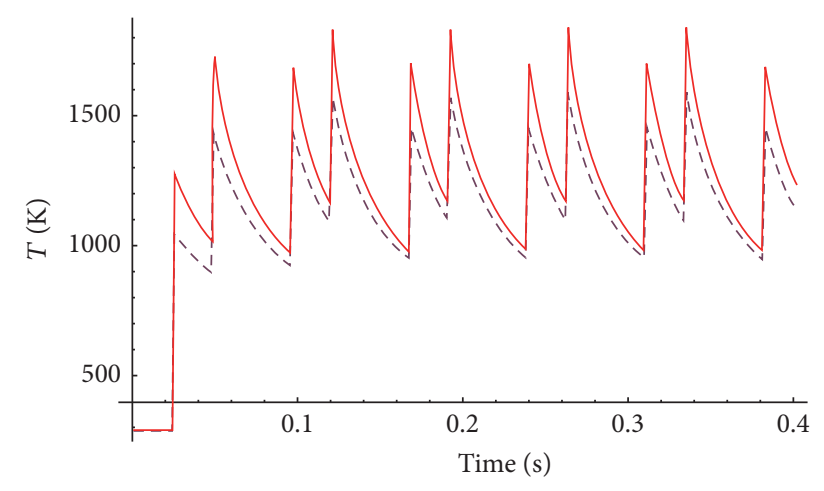

FIGURE 14: Evolution of maximum foil temperatures [K] at the $\mathrm{H}^{-}$ spot peak (dashed) and the combined $\mathrm{H}^{-}$and circulating protons peak (red). Two injections into the accumulator per linac pulse.

modifications, provided the SNS-like injection lattice is kept for the ESSnuSB accumulator design.

The injection process is presently being studied by tracking particles from the linac using the ESS linac beam parameters derived from simulations. The idea is to "paint" the phase space at injection in a way such as to minimize space charge effects, while keeping a limited beam size. After the beam pulse (550 turns) is injected, the beam is extracted in one turn to the target.

Stripping foil temperatures were computed using several different methods $[29,34]$ giving very similar results. Calculations are based on ground-laying work made for the SNS injection [35]. Minimizing the number of foil passes of the circulating beam requires a local increase of its transverse size. Promising results were obtained by changing the injection optics to increase the linac spot size on the foil. A maximum temperature of $1550 \mathrm{~K}$ on the foil was obtained. For this case the average number of foil passes of the circulating beam was found to be about 4.8 and the emittance of the circulating beam was $200 \pi(95 \%)$ normalized. The maximum temperature on the foil for SNS operation does not exceed $1600 \mathrm{~K}$. Further simulations show that, even for $100 \pi$ (95\%) normalized emittance, foil temperatures can be kept at levels likely to be acceptable, with the hottest spots at $1650 \mathrm{~K}$ [36].

The case of filling the ring only twice with a linac pulse of $1.5 \mathrm{~ms}$ each time, instead of four times with a pulse of $0.7 \mathrm{~ms}$, has been calculated. This configuration would reduce the linac pulsing frequency or the number of stacked accumulators, respectively. Peak temperatures of $1560 \mathrm{~K}$ for the $\mathrm{H}^{-}$beam alone and $1850 \mathrm{~K}$ for the sum of the effects of $\mathrm{H}^{-}$and $\mathrm{H}^{+}$beams are reached. The maximum space charge tune shifts remain below -0.16 , which is a clearly acceptable value. The temperature profiles on the foil resulting from this calculation are shown in Figure 14. The normalized emittance of the fully injected beam is in this case $200 \pi$. However, the final beam emittance in the ring will be constrained by the requirements to have efficient extraction of the beam from the accumulator and efficient transport of the beam from the accumulator, through the switchyard [37] up to the four targets, still keeping the temperature of the foil within limits.

\section{The Target Station}

The main elements of the secondary neutrino beam are the target station with the four-horn/target systems to produce and focus the charged mesons, the $25 \mathrm{~m}$ long decay tunnel to produce the neutrinos from the meson decay, and the beam dump to absorb the undecayed mesons. The apparatus will be heavily shielded with iron and concrete due to the high radiation produced.

The target station design and the short pion-decay tunnel have already been studied for the Super Beam within the EUROnu [10] project with further optimizations for the ESSnuSB project [38]. In order to mitigate the detrimental effects of the high power beam, four targets and horns are needed; each target will be hit by a $1.25 \mathrm{MW}$ proton beam. The target is placed inside the inner conductor of the horn for maximum particle collection while the shape of the inner conductor is optimized such as to produce the best possible neutrino beam for the discovery of $\mathrm{CP}$ violation [38].

The target is made of a bed of $3 \mathrm{~mm}$ diameter titanium spheres packed in an about $530 \mathrm{~mm}$ long canister with $30 \mathrm{~mm}$ diameter and cooled with helium gas. This type of target can be more efficiently cooled and stress development on the spheres is less than on the usual monolithic or segmented solid targets used in the past. Thermal modeling has shown that it is possible to cool the packed-bed target in a way to accept more than $1 \mathrm{MW}$ beam power [38].

For the EUROnu Super Beam, a $350 \mathrm{kA}$ pulsed power supply running at $50 \mathrm{~Hz}$ frequency was proposed for the four horns [39]. For ESSnuSB, additional studies have shown that modifications could be made in order to comply with $56 \mathrm{~Hz}$ and other possible pulsing scenarios [40]. To maintain acceptable stress and deformation on the conductors of the horn that are produced by the pulsed current and the particlecrossing, water jets have been proposed for cooling. The water jet system is able to maintain a constant temperature along the conductors. Preliminary fatigue studies on such a setup, taking into account the thermodynamical analyses performed for the horns, indicate that each horn can withstand a year $\left(10^{8}\right.$ pulses) of operation [38].

Experiments, including vibration tests as well as tests of the cooling capacity of the proposed system, can be made at the high intensity proton irradiation facility at CERN [41].

The region surrounding the four-horn/target system is shielded such as to produce less than $10 \mu \mathrm{Sv} / \mathrm{h}$ prompt effective dose on the areas above, for example, the top floor and the power supply unit room. The region surrounding the decay tunnel and the beam dump is shielded such that the activation of the rock complies with the ESS safety rules. In the preliminary radiation studies of EUROnu a depth of $10 \mathrm{~m}$ inside the molasse rock was considered.

The neutrino beam has to point down from the horizontal plane at ESS by $2.67^{\circ}$ (46.6 mrad) to get to the Garpenberg mine at $540 \mathrm{~km}$ distance, where the Far Detector will be placed. From this follows the fact that the target station will be at $25 \mathrm{~m}$ depth, giving space to proton beam extraction equipment and the switchyard. The SPL Super Beam target station was designed to be situated at $18 \mathrm{~m}$ depth; thus $25 \mathrm{~m}$ 
depth for the ESSnuSB should be compatible with radiation safety and civil engineering constraints.

\section{Design Considerations for the Near Detector}

In order to achieve the required experimental sensitivity it is important to construct a Near Detector (ND) close to the neutrino production point. The ND should be able to measure the flux of each neutrino species in the beam, directly after production, to precision better than $5 \%$. As the electron neutrino content in the beam is $0.3 \%$, the ND needs to be able to separate Charged Current (CC) muon and electron neutrino events at a level better than $10^{-4}$. The preliminary ND design suggested here resulted from a series of simulations. The proposal is to use a cylindrical water Cherenkov detector with a radius, $R_{\mathrm{ND}}=5 \mathrm{~m}$, and a length, $L_{\mathrm{ND}}=10 \mathrm{~m}$, positioned with its central axis aligned with the direction of the neutrino beam, $z_{\mathrm{ND}}=500 \mathrm{~m}$, from the target station. On the outside it is covered with scintillator plates to veto atmospheric muons and to reject events not fully contained in the detector. Further details about the study of this ND design can be found in [42].

The input to the study was a neutrino beam profile obtained using FLUKA 2011.xx (version 2014) [43] and GEANT 3.21 [44]. The calculation was made for a proton beam energy of $2.0 \mathrm{GeV}$. The neutrino energy distribution, for the case where the horn focuses positively charged particles, is shown by the gray-shaded histogram in Figure 4. The neutrinos from this beam were used to simulate neutrino interaction events in water using the GENIE toolkit [45]. The angular acceptance of the Far Detector (FD) is $\pm 0.1 \mathrm{mrad}$, while for the ND it is $\pm 10 \mathrm{mrad}$. As the ND provides the baseline for the FD measurements, it is important that the neutrino energy spectrum and the flavour composition of the beam are not significantly different for the two detectors. It was verified that when the angle with respect to the beam axis of the produced neutrinos varies from 0 to $10 \mathrm{mrad}$, the change in neutrino flavour composition and energy distribution is indeed negligible. At an ND distance, $z_{\mathrm{ND}}=$ $500 \mathrm{~m}$, with a radius, $R_{\mathrm{ND}}=5 \mathrm{~m}$, and length, $L_{\mathrm{ND}}=10 \mathrm{~m}$, the muon neutrino CC event rate would be $260 \mathrm{~s}^{-1}$ and the electron neutrino CC event rate $1 \mathrm{~s}^{-1}$.

For ESSnuSB neutrino energies the muons resulting from $\mathrm{CC}$ collisions travel less than $3 \mathrm{~m}$ in water (the CC electrons travel shorter) before they stop radiating Cherenkov light. This implies that most of the events will be fully contained within the cylindrical ND water volume given above. To explore the conditions for the event reconstruction, an event sample was produced, containing idealized neutrino CC event products in a simplified detector environment. Each event in this sample was initiated with a charged lepton with a predetermined kinetic energy and momentum along the $z$-axis. A photodetector wall was placed in the $x-y$ plane at $z=5 \mathrm{~m}$, where each photon was registered on impact. From the timing and position of the detected photons one can determine the position of the initial vertex, the direction of the charged lepton, its flavour, and its energy. An algorithm, to determine the event vertex coordinates, was developed that iteratively investigates a number of coordinate sets in the detector using the timing and positions of the detected photons. Using photodetectors with time resolution in the order of $0.1 \mathrm{~ns}$ and position resolution in the order of $1 \mathrm{~cm}$, this algorithm can locate the vertex with an accuracy of a few centimeters. Further information concerning the principles for event reconstruction in a water Cherenkov detector is given in, for example, [46].

The simulations also showed that the energy of the lepton could be estimated from the number of detected photons, $N_{\gamma}$, which is assumed to be roughly proportional to $E_{\text {kin }}-$ $E_{\mathrm{ChTh}}$, where $E_{\text {kin }}$ is the lepton kinetic energy and $E_{\mathrm{ChTh}}$ is the threshold energy for Cherenkov light production. The lepton flavour identification was done by examining the fuzziness of the edge of the produced Cherenkov ring. For this purpose an algorithm was developed, based on a modified version of the circular Hough transform [47] that used filled circles instead of ring contours. Lepton flavour identification was done using this algorithm by fitting a "muon ring" and an "electron ring" to the detected photons of each event, to determine which flavour was most probable to have produced the detector response. The algorithm looks at several parameters and awards each event a number of $\mu$ votes and $e$-votes. By applying cuts to these votes the lepton flavour is determined. This first trial identification algorithm yielded a misidentification rate of $0.3 \%$. This is still larger than required and further work is thus needed. All misidentified events were muons that had been identified as electrons and were mainly found among lower energy events. Details of the algorithm used and the results obtained can be found in [42].

\section{Project Time Schedule}

The aim of the presently ongoing ESSnuSB Design Study is to produce a Conceptual Design Report by 2018 to be followed by a Technical Design Report by 2020. This assumes that adequate financial support for the Design Study can be secured. Taking into account technical constraints only, the upgrade of the linac and the construction of the accumulator, of the neutrino target station, of the Near Detector, and of the Far Detector could start by 2021. The ESSnuSB buildup period is estimated to take some 7 years (dominated by the construction of the Far Detector) leading up to start of data taking at the earliest by 2027. Taking other funding and organizational constraints into account, 2030 is a more probable date for start of data taking.

A crucial prerequisite for this time schedule is that the comparatively modest modifications of the linac that are described at the end of Section 3 are made during the linac build-up period 2017-2023. To introduce the same modifications once the linac is running for spallation neutron production will be disruptive to the neutron experiments, will take more time, and will be more costly.

\section{Summary and Conclusions}

Leptonic CP violation potentially plays a crucial role for the explanation of matter-antimatter asymmetry in the Universe. The prime opportunity to discover and measure leptonic $\mathrm{CP}$ violation is offered by future Super Beam experiments 
studying the $v_{\mu} \rightarrow v_{e}$ oscillation. Following the discovery in 2012 of a large value of the neutrino mixing angle $\theta_{13}$, analytical calculations show that a higher sensitivity to $\mathrm{CP}$ violation is obtained by measuring at the second neutrino oscillation maximum, as compared to the first.

This fact has been shown in this paper by comparing directly the sensitivities to $\mathrm{CP}$ violation of experimental observables, such as the number of detected electron neutrinos and the normalized electron neutrino-antineutrino asymmetry at the first and the second maximum, respectively. The higher sensitivity, using the same conservative assumptions for systematic errors, at the second maximum is also apparent from comparisons previously made between different Super Beam projects on the basis of global simulation calculations. In view of the very high neutrino beam intensity required for measurements at the second maximum, the uniquely high power of the ESS proton driver represents a significant advantage for such measurements.

ESSnuSB is currently the only Super Beam experiment which concentrates on taking its data at the second oscillation maximum. Global calculations show that, taking data with ESSnuSB with a $2.0 \mathrm{GeV}(2.5 \mathrm{GeV})$ ESS proton beam during 10 years using the MEMPHYS Megaton water Cherenkov detector placed in the $1200 \mathrm{~m}$ deep Garpenberg mine at the second maximum, $540 \mathrm{~km}$ from ESS, the coverage of the range of possible $\mathrm{CP}$ violating angle values is $56 \%$ (65\%) assuming the neutrino hierarchy to be known and the systematic errors to be those shown in the "SB Opt." column of Table 3, in which the neutrino flux error is $5 \%$ and the neutrino cross section error is $10 \%$.

The generation of the neutrino Super Beam using the ESS linac will require acceleration of $\mathrm{H}^{-}$pulses. These pulses will be interleaved with the proton pulses accelerated for neutron production in such a way that the linac will deliver a $5 \mathrm{MW} \mathrm{H}^{-}$beam simultaneously with the $5 \mathrm{MW}$ proton beam. The $\mathrm{H}^{-}$beam will be injected into a $376 \mathrm{~m}$ circumference accumulator ring, stripping off the two electrons of each $\mathrm{H}^{-}$ion at the entrance to the ring, by multiturn injection and ejected from the ring by single turn extraction, thereby obtaining compression of the pulse length from $2.86 \mathrm{~ms}$ to $1.3 \mu$ s. The ejected $\mathrm{H}^{-}$beam will be guided to a neutrino target with a surrounding horn-type hadron collector, downstream of which there is a $25 \mathrm{~m}$ long pion-decay tunnel. The very high current required in the horn cannot be maintained for more than a few $\mu$ s which is what necessitates the strong compression of the linac pulse.

The accumulator ring and the target station can be installed underground on the ESS site without significant interference with the linac construction and operation. The introduction of $\mathrm{H}^{-}$pulses in the linac and the doubling of the linac average power can to a large extent be made after the completion of the linac, as presently designed, without major interference with the linac operation provided, however, that certain comparatively modest, but crucial, preparative modifications are made to the linac already during its buildup phase 2017-2023.

The accumulator ring, which will contain an exceptionally high number of protons, represents a challenging design task, in particular its injection scheme and its ring lattice and collimation. In order to somewhat reduce the complexity of the task, the number of protons per pulse can be reduced by using one of two alternative schemes: by injecting more and shorter $\mathrm{H}^{-}$pulses in the compressor ring or by splitting the long $\mathrm{H}^{-}$pulses up on more than one compressor ring. Design work of an accumulator ring receiving a sequence of pulses after each $2.86 \mathrm{~ms}$ long proton pulse in the linac and of an injection stripping scheme, based on foil stripping, is ongoing. This study will give us an indication of how many particles that can be stored, which will give the number of linac pulses or the number of accumulators that will be needed to handle the full linac beam and send it to the four targets.

The Near Detector plays a crucial role for the determination of the neutrino flux needed in the evaluation of the Far Detector data. The simulation of a cylindrical water Cherenkov detector, $10 \mathrm{~m}$ long and $5 \mathrm{~m}$ in radius located $500 \mathrm{~m}$ downstream of the target, has been used to study how such a detector could match the requirements. Methods for the determination of the position of the initial CC vertex, the direction of the charged lepton, its flavour, and its energy have been designed and evaluated. The results obtained so far are encouraging but need to be worked on further to satisfy the demanding requirement of not more than $5 \%$ systematic error in the Far Detector measurements.

The current ESSnuSB Design Study is foreseen to lead to a CDR in 2018 and to a TDR in 2020. Taking into account technical constraints only, construction of all parts could start in 2021 and be completed at the earliest by 2027.

\section{Competing Interests}

The authors declare that they have no competing interests.

\section{Acknowledgments}

The authors are grateful to E. Fernandez-Martinez and P. Coloma who have contributed with calculations and plots for the physics part of this paper, to F. Gerigk and E. Montesinos for their study and report of the modifications of the ESS linac that are required to allow simultaneous acceleration of $\mathrm{H}^{+}$ and $\mathrm{H}^{-}$ions at an average power of $5+5 \mathrm{MW}$, and to $\mathrm{B}$. Holzer and $\mathrm{B}$. Goddard for giving help with aspects related to the accumulator design.

\section{References}

[1] K. Abe, N. Abgrall, Y. Ajima et al., "Indication of electron neutrino appearance from an accelerator-produced off-axis muon neutrino beam," Physical Review Letters, vol. 107, no. 4, Article ID 041801, 2011.

[2] Y. Abe, C. Aberle, T. Akiri et al., "Indication of reactor $\bar{v}_{e}$ disappearance in the double chooz experiment," Physical Review Letters, vol. 108, Article ID 131801, 2012.

[3] J. K. Ahn, S. Chebotaryov, J. H. Choi et al., "Observation of reactor electron antineutrinos disappearance in the reno experiment," Physical Review Letters, vol. 108, no. 19, Article ID 191802, 2012. 
[4] F. P. An, J. Z. Bai, A. B. Balantekin et al., "Observation of electron-antineutrino disappearance at daya bay," Physical Review Letters, vol. 108, no. 17, Article ID 171803, 2012.

[5] M. V. Diwan, D. Beavis, M.-C. Chen et al., "Very long baseline neutrino oscillation experiments for precise measurements of mixing parameters and $C P$ violating effects," Physical Review D, vol. 68, no. 1, Article ID 012002, 2003.

[6] S. Peggs, R. Kreier, C. Carlile et al., Eds., ESS Technical Design Report, ESS-doc-274, ESS, Lund, Sweden, 2013, http://eval.esss .lu.se/cgi-bin/public/DocDB/ShowDocument?docid=274.

[7] "Long-baseline neutrino facility (LBNF) and deep underground neutrino experiment (DUNE)," Conceptual Design Report, 2015.

[8] K. Abe, H. Aihara, C. Andreopoulos et al., "Physics potential of a long-baseline neutrino oscillation experiment using a J-PARC neutrino beam and Hyper-Kamiokande," Progress of Theoretical and Experimental Physics, vol. 2015, Article ID 053C02, 2015.

[9] E. Baussan, M. Blennow, M. Bogomilov et al., "A very intense neutrino super beam experiment for leptonic $\mathrm{CP}$ violation discovery based on the European spallation source linac," Nuclear Physics B, vol. 885, pp. 127-149, 2014.

[10] T. R. Edgecock, O. Caretta, T. Davenne et al., "High intensity neutrino oscillation facilities in Europe," Physical Review Accelerators and Beams, vol. 16, no. 2, Article ID 021002, 18 pages, 2013.

[11] F. Gerigk, S. Atieh, I. Aviles Santillana et al., Conceptual Design of the Low-Power and High-Power SPL: A Superconducting $H^{-}$ Linac at CERN, CERN, Geneva, Switzerland, 2014.

[12] O. Brunner, S. Calatroni, E. Ciapala et al., "Assessment of the basic parameters of the cern superconducting proton linac," Physical Review Accelerators and Beams, vol. 12, no. 7, Article ID 070402, 24 pages, 2009.

[13] E. Baussan, J. Bielski, C. Bobeth et al., "Neutrino super beam based on a superconducting proton linac," Physical Review Accelerators and Beams, vol. 17, no. 3, Article ID 031001, 26 pages, 2014.

[14] L. Agostino, M. Buizza-Avanzini, M. Dracos et al., "Study of the performance of a large scale water-Cherenkov detector (MEMPHYS)," Journal of Cosmology and Astroparticle Physics, vol. 2013, article 024, 2013.

[15] F. Gerigk and E. Montesinos, "Recommended modifications of the ESS baseline architecture for ESSnuSB," Tech. Rep. CERN-ACC-NOTE-2015-0040, CERN, Geneva, Switzerland, 2015, http://cds.cern.ch/record/2110681.

[16] D. McGinnis, M. Lindroos, and R. Miyamoto, "European spallation source afterburner concept," in Proceedings of the 4th International Particle Accelerator Conference (IPAC '13), pp. 3924-3926, Shanghai, China, May 2013.

[17] T. Patzak, "LAGUNA and LAGUNA-LBNO: future megaton neutrino detectors in Europe," Nuclear Instruments and Methods in Physics Research Section A: Accelerators, Spectrometers, Detectors and Associated Equipment, vol. 695, pp. 184-187, 2012.

[18] P. Coloma, P. Huber, J. Kopp, and W. Winter, "Systematic uncertainties in long-baseline neutrino oscillations for large $\theta_{13}$, Physical Review D, vol. 87, no. 3, Article ID 033004, 12 pages, 2013.

[19] P. Coloma and E. Fernandez-Martinez, "Optimization of neutrino oscillation facilities for large $\theta_{13}$," Journal of High Energy Physics, vol. 2012, article 89, 2012.

[20] M. Bass, M. Bishai, D. Cherdack et al., "Baseline optimization for the measurement of $\mathrm{CP}$ violation, mass hierarchy, and $\theta_{23}$ octant in a long-baseline neutrino oscillation experiment," Physical Review D, vol. 91, no. 5, Article ID 052015, 2015.

[21] R. Aleksan, P. Braun-Munzinger, P. Chomaz et al., "Physics briefing book: input for the strategy group to draft the update of the European strategy for particle physics," Tech. Rep. CERNESG-005, CERN, Geneva, Switzerland, 2013, Open Symposium held in Cracow from 10th to 12th of September 2012.

[22] C. Bronner, "T2K oscillation results," in Proceedings of the 16th International Workshop on Neutrino Factories and Future Neutrino Beam Facilities (NUFACT '14), Proceedings of Science, Glasgow, UK, August 2014.

[23] S. Parke, "Neutrinos: theory and phenomenology," Physica Scripta T, vol. 158, Article ID 014013, 2013.

[24] P. Huber, M. Lindner, and W. Winter, "Simulation of longbaseline neutrino oscillation experiments with GLoBES: (General Long Baseline Experiment Simulator)," Computer Physics Communications, vol. 167, no. 3, pp. 195-202, 2005.

[25] P. Huber, J. Kopp, M. Lindner, M. Rolinec, and W. Winter, "New features in the simulation of neutrino oscillation experiments with GLoBES 3.0: General Long Baseline Experiment Simulator," Computer Physics Communications, vol. 177, no. 5, pp. 432438, 2007.

[26] A. de Gouvea, K. Pitts, K. Scholberg et al., "Working group report: neutrinos," in Proceedings of the Community Summer Study: Snowmass on the Mississippi (CSS '13), Minneapolis, Minn, USA, August 2013.

[27] SNS design manual, 2003, https://neutrons.ornl.gov/sns.

[28] A. J. Jason, D. W. Hudgings, and O. B. van Dyck, "Neutralization of $\mathrm{H}^{-}$beams by magnetic stripping," IEEE Transactions on Nuclear Science, vol. 28, no. 3, pp. 2703-2706, 1981.

[29] E. Wildner, J. Jonnerby, J.-P. Koutchouk et al., "The accumulator of the ESSnuSB for neutrino production," in Proceedings of the 5th International Particle Accelerator Conference (IPAC '14), pp. 2245-2247, Dresden, Germany, June 2014.

[30] E. Bouquerel, M. Dracos, and F. Osswald, "Preliminary design of a $4 \mathrm{MW}$ proton beam switchyard for a neutrino super beam production facility," in Proceedings of the 4th International Particle Accelerator Conference (IPAC '13), pp. 1880-1882, Shanghai, China, May 2013.

[31] M. Olvegård, A. Bhattacharyya, D. Dancila et al., "Progress at the FREIA laboratory," in Proceedings of the 6th International Particle Accelerator Conference (IPAC '15), pp. 3072-3075, Richmond, VA, USA, May 2015.

[32] M. Fitterer, Design study of the large hadron electron collider and a rapid cycling synchrotron as alternative to the PS booster upgrade at CERN [Ph.D. thesis], Karlsruhe University, Karlsruhe, Germany, 2013.

[33] A. V. Aleksandrov, T. V. Gorlov, V. V. Danilov, and Y. Liu, "Status of laser stripping at the SNS," in Proceedings of the 24th Particle Accelerator Conference (PAC '11), C110328, pp. 2035-2037, New York, NY, USA, March-April 2011.

[34] M. Martini, "Modelling accumulator stripper foil heating for ESSNUSB facility," Tech. Rep. CERN-ACC-NOTE-2015-0005, CERN, Geneva, Switzerland, 2015.

[35] M. A. Plum, J. Holmes, R. W. Shaw, and C. S. Feigerle, "SNS stripper foil development program," Nuclear Instruments and Methods in Physics Research Section A: Accelerators, Spectrometers, Detectors and Associated Equipment, vol. 590, no. 1-3, pp. 43-46, 2008.

[36] E. Wildner, J. Jonnerby, J. P. Koutchouk et al., "The accumulator of the ESSnuSB for neutrino production," in Proceedings of the 
6th International Particle Accelerator Conference (IPAC '15), pp. 3942-3944, Richmond, Va, USA, May 2015.

[37] E. Bouquerel, "Design status of the ESS vSB switchyard," in Proceedings of the 6th International Particle Accelerator Conference (IPAC '15), pp. 125-127, Richmond, VA, USA, May 2015.

[38] E. Baussan, J. Bielski, C. Bobeth et al., "Neutrino super beam based on a superconducting proton linac," Physical Review Special Topics-Accelerators and Beams, vol. 17, no. 3, Article ID 031001, 26 pages, 2014.

[39] E. Baussan, E. Bouquerel, M. Dracos et al., "Study of the pulse power supply unit for the four-horn system of the CERN to Fréjus neutrino super beam," Journal of Instrumentation, vol. 8, no. 7, Article ID T07006, 2013.

[40] N. Vassilopoulos, "ESS $\nu$ SB: update on secondary beam studies," in Proceedings of the 16th International Workshop on Neutrino Factories and Future Neutrino Beam Facilities (NUFACT '14), Proceedings of Science, Glasgow, UK, August 2014.

[41] N. Charitonidis, I. Efthymiopoulos, A. Fabich, M. Meddahi, and E. Gianfelice-Wendt, "Status and planned experiments of the HiRadMat pulsed beam material test facility at CERN SPS," in Proceedings of the 6th International Particle Accelerator Conference (IPAC '15), WEPMN072, Richmond, Va, USA, May 2015.

[42] A. Burgman, A neutrino detector design-a simulation study on the design of the cherenkov near detector of the proposed ESS $\nu S B$ [M.S. thesis], Lund University, Lund, Sweden, 2015.

[43] A. Ferrari, P. R. Sala, A. Fassò, and J. Ranft, "FLUKA: a multiparticle transport code (program version 2005)," Tech. Rep. CERN-2005-10; INFN/TC 05/11; SLAC-R-773, CERN, Geneva, Switzerland, 2005.

[44] S. Agostinelli, J. Allison, K. Amako, J. Apostolakis, H. Araujo, and P. Arce, "Geant4-a simulation toolkit," Nuclear Instruments and Methods in Physics Research A: Accelerators, Spectrometers, Detectors and Associated Equipment, vol. 506, no. 3, pp. 250-303, 2003.

[45] C. Andreopoulos, A. Bell, D. Bhattacharya et al., "The GENIE neutrino Monte Carlo generator," Nuclear Instruments and Methods in Physics Research A, vol. 614, no. 1, pp. 87-104, 2010.

[46] K. Abe, Y. Hayato, T. Iida et al., "Solar neutrino results in SuperKamiokande-III," Physical Review D, vol. 83, no. 5, Article ID 052010, 19 pages, 2010.

[47] R. O. Duda and P. E. Hart, "Use of the Hough transformation to detect lines and curves in pictures," Communications of the ACM, vol. 15, no. 1, pp. 11-15, 1972. 

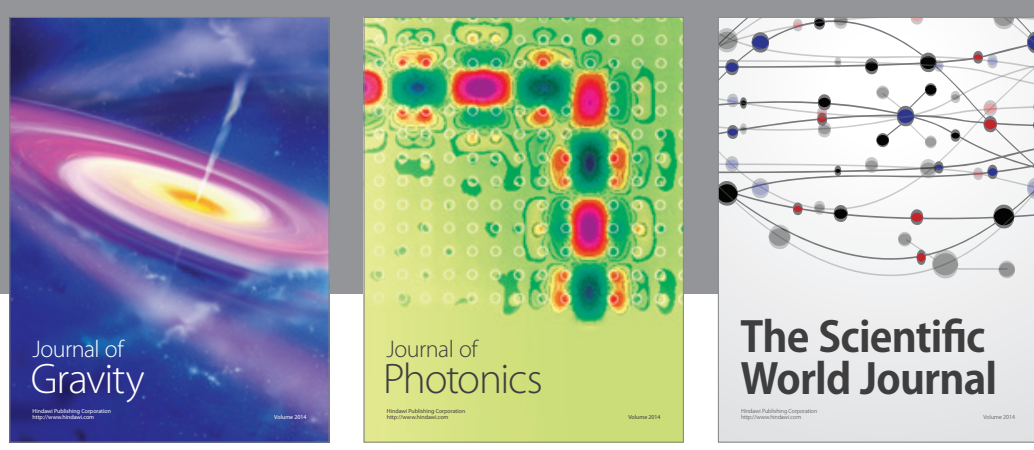

The Scientific World Journal
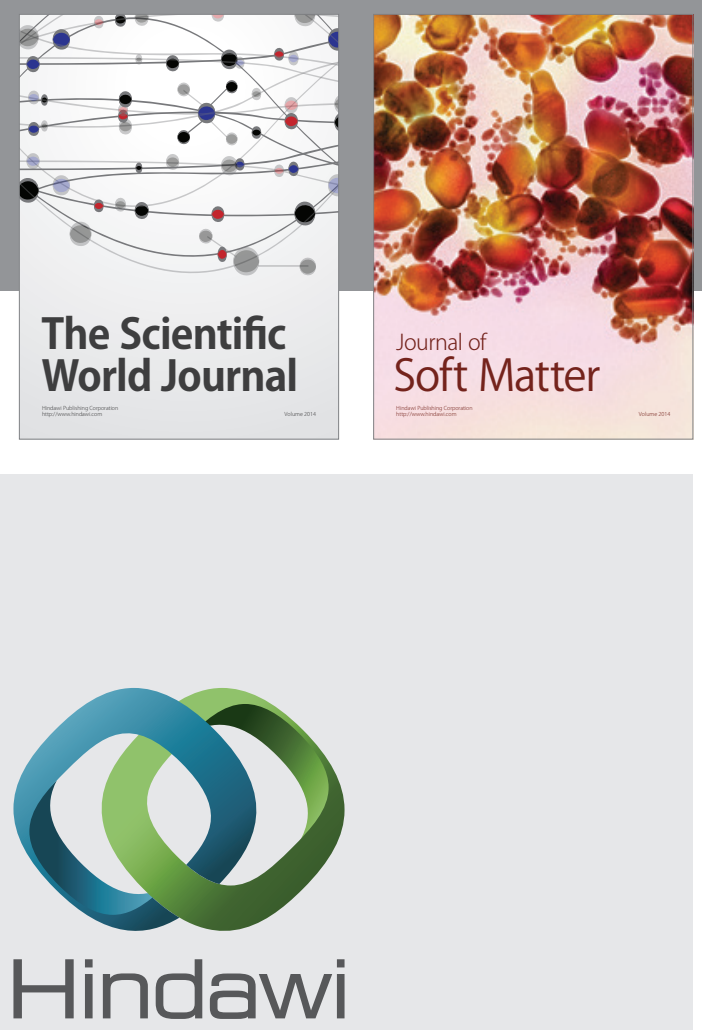

Submit your manuscripts at

http://www.hindawi.com

nternational Journal of

Statistical Mechanics
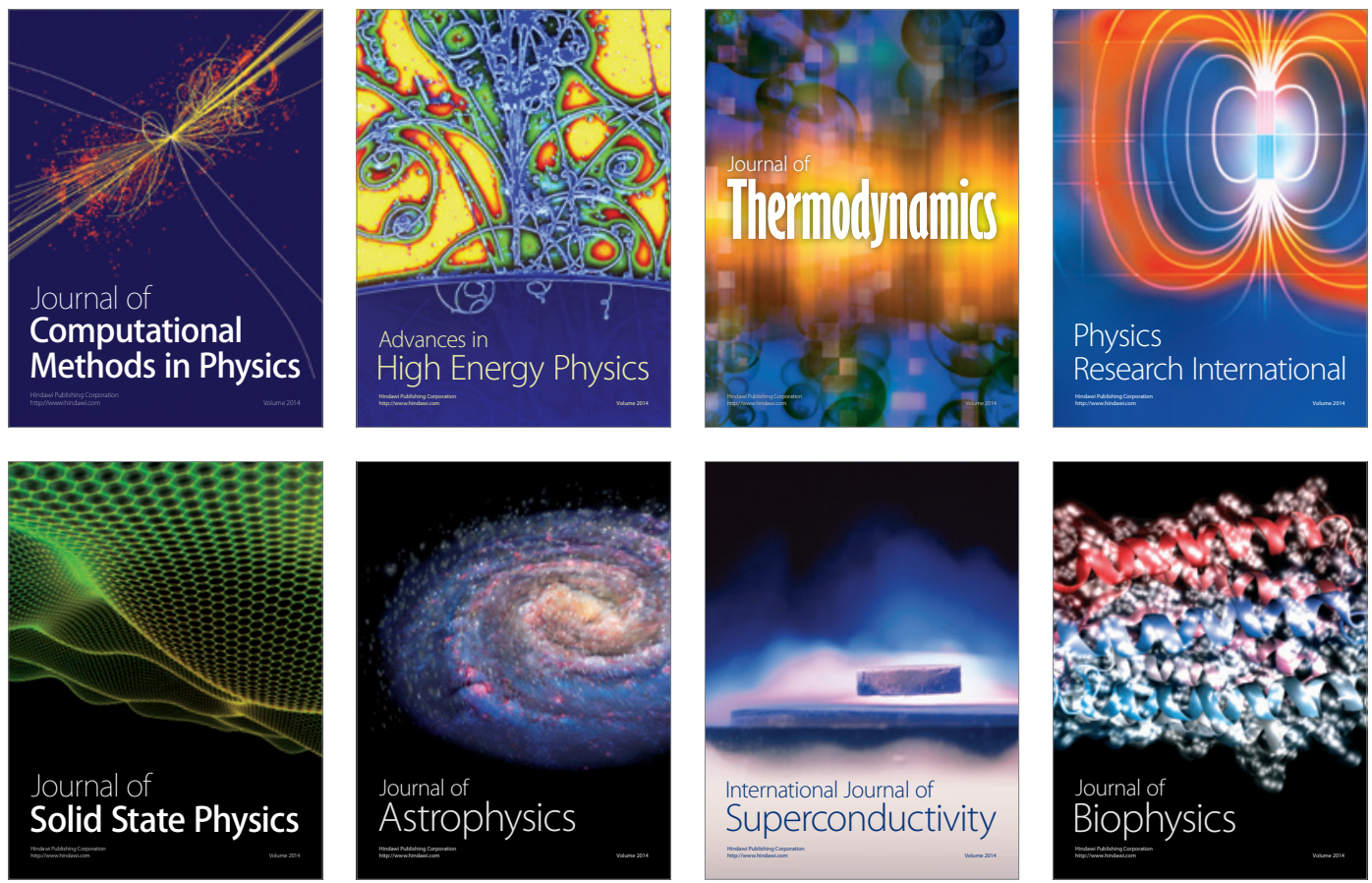
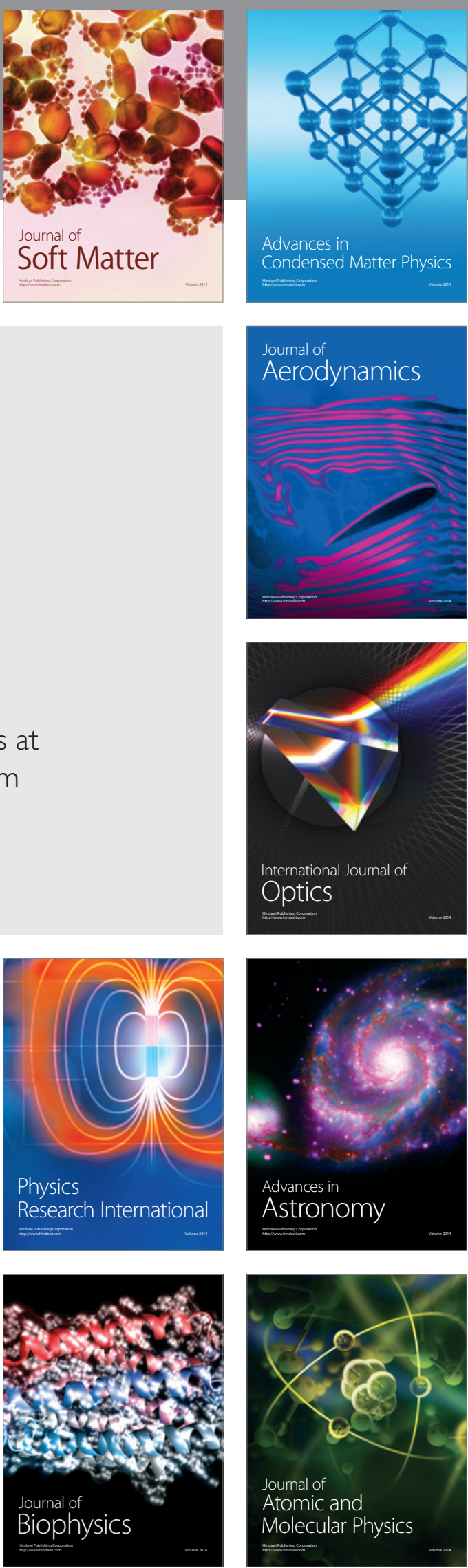